Provided for non-commercial research and education use. Not for reproduction, distribution or commercial use.

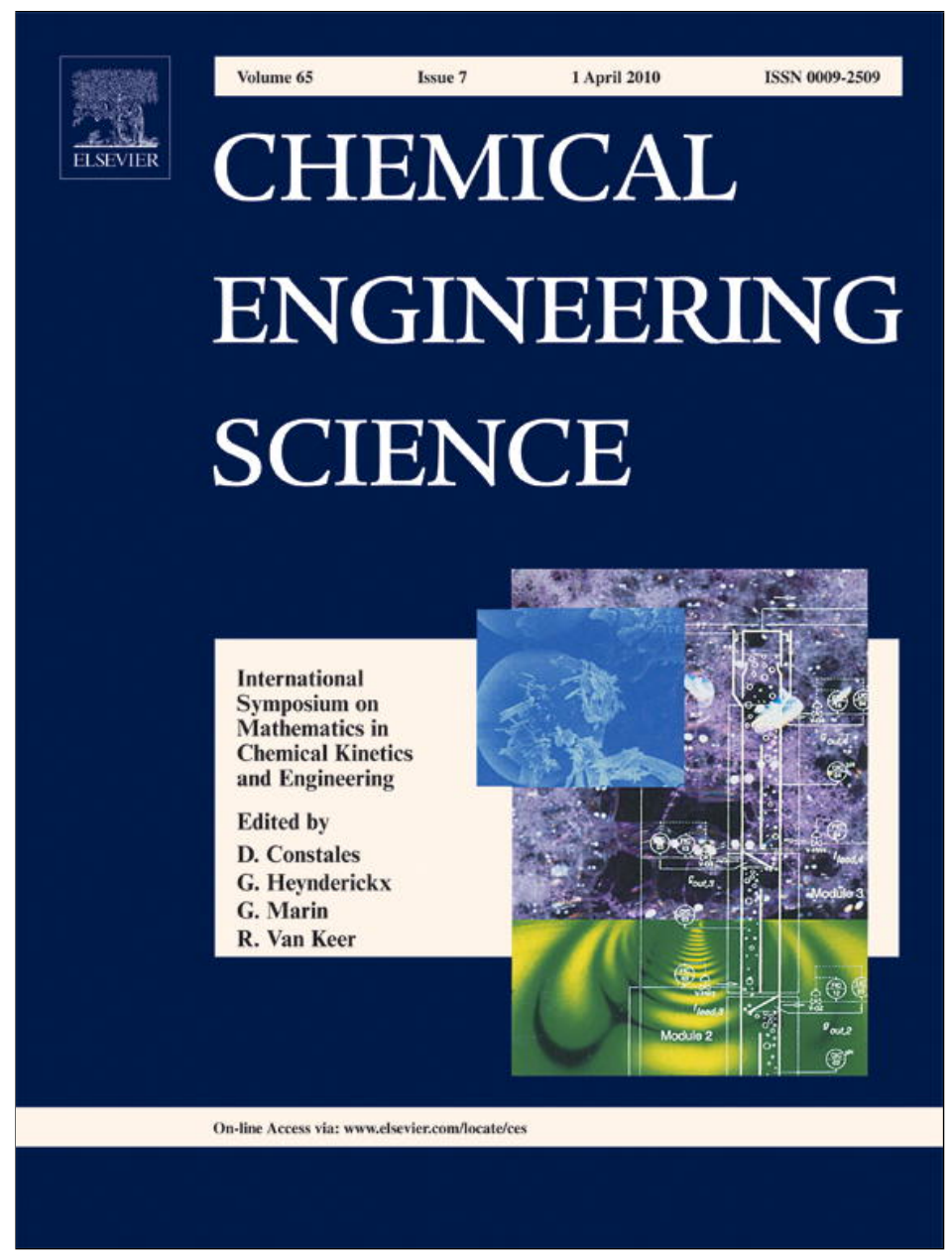

This article appeared in a journal published by Elsevier. The attached copy is furnished to the author for internal non-commercial research and education use, including for instruction at the authors institution and sharing with colleagues.

Other uses, including reproduction and distribution, or selling or licensing copies, or posting to personal, institutional or third party websites are prohibited.

In most cases authors are permitted to post their version of the article (e.g. in Word or Tex form) to their personal website or institutional repository. Authors requiring further information regarding Elsevier's archiving and manuscript policies are encouraged to visit:

http://www.elsevier.com/copyright 


\title{
Asymptotology of chemical reaction networks
}

\author{
A.N. Gorban ${ }^{\mathrm{a}, *}$, O. Radulescu $^{\mathrm{b}}$, A.Y. Zinovyev ${ }^{\mathrm{c}}$ \\ a University of Leicester, LE1 7RH Leicester, UK \\ ${ }^{\mathrm{b}}$ IRMAR, UMR 6625, University of Rennes 1, Campus de Beaulieu, 35042 Rennes, France \\ ${ }^{\mathrm{c}}$ Institut Curie, U900 INSERM/Curie/Mines ParisTech, 26 rue d'Ulm, F75248 Paris, France
}

\section{A R T I C L E I N F O}

\section{Article history:}

Received 2 April 2009

Received in revised form

13 August 2009

Accepted 1 September 2009

Available online 9 September 2009

Keywords:

Reaction network

Asymptotology

Dominant system

Limiting step

Multiscale asymptotic

Model reduction

\begin{abstract}
A B S T R A C T
The concept of the limiting step is extended to the asymptotology of multiscale reaction networks. Complete theory for linear networks with well separated reaction rate constants is developed. We present algorithms for explicit approximations of eigenvalues and eigenvectors of kinetic matrix. Accuracy of estimates is proven. Performance of the algorithms is demonstrated on simple examples. Application of algorithms to nonlinear systems is discussed.
\end{abstract}

(c) 2009 Elsevier Ltd. All rights reserved.

\section{Introduction}

Most of mathematical models that really work are simplifications of the basic theoretical models and use in the backgrounds an assumption that some terms are big, and some other terms are small enough to neglect or almost neglect them. The closer consideration shows that such a simple separation on "small" and "big" terms should be used with precautions, and special culture was developed. The name "asymptotology" for this direction of science was proposed by Kruskal (1963), but fundamental research in this direction are much older, and many fundamental approaches were developed by I. Newton (Newton polyhedron, and many other things).

Following Kruskal (1963), asymptotology is "the art of describing the behavior of a specified solution (or family of solutions) of a system in a limiting case. ... The art of asymptotology lies partly in choosing fruitful limiting cases to examine... . The scientific element in asymptotology resides in the non-arbitrariness of the asymptotic behavior and of its description, once the limiting case has been decided upon".

\footnotetext{
* Corresponding author.

E-mail addresses: ag153@le.ac.uk (A.N. Gorban), ovidiu.radulescu@uni v-rennes1.fr (O. Radulescu), andrei.zinovyev@curie.fr (A.Y. Zinovyev).
}

Asymptotic behavior of rational functions of several positive variables $k_{i}>0$ gives us a toy-example. Let

$R\left(k_{1}, \ldots, k_{n}\right)=P\left(k_{1}, \ldots, k_{n}\right) / Q\left(k_{1}, \ldots, k_{n}\right)$

be such a function and $P, Q$ be polynomials. To derive fruitful limiting cases we consider logarithmic straight lines $\ln k_{i}=\theta_{i} \xi$ and study asymptotical behavior of $R$ for $\xi \rightarrow \infty$. In this asymptotics, for almost every vector $\left(\theta_{i}\right)$ (outside several hyperplanes) there exists such a dominant monomial $R_{\infty}(k)=A \prod_{i} k_{i}^{\alpha_{i}}$ that $R=R_{\infty}+o\left(R_{\infty}\right)$. The function that associates a monomial with vector $\left(\theta_{i}\right)$ is piecewise constant: it is constant inside some polyhedral cones.

Implicit functions given by equations which depend on parameters provide plenty of more interesting examples, especially in the case when the implicit function theorem is not applicable. Some analytical examples are presented by Andrianov and Manevitch (2002) and White (2006). Introduction of algebraic backgrounds and special software is provided by Greuel and Pfister (2002).

For a difficult problem, analysis of eigenvalues and eigenvectors of non-symmetric matrices, Vishik and Ljusternik (1960) studied asymptotic behavior of spectra and spectral projectors along the logarithmic straight lines in the space of matrices. This analysis was continued by Lidskii (1965).

We study networks of linear reactions. For a linear system with reaction rate constants $k_{i}$ all the dynamical information is contained in eigenvalues and eigenvectors of the kinetic matrix 
or, more precisely, in its transformation to the Jordan normal form. It is computationally expensive task to find this transformation for a non-symmetric matrix which is usually stiff (Golub and VanLoan, 1996). Moreover, the answer could be very sensitive to the errors in constants $k_{i}$. Nevertheless, it appears that stiffness can help us to find a robust approximation, and in the limit when all constants are very different (well-separated constants) the asymptotical behavior of eigenvalues and eigenvectors follow simple explicit expressions. Analysis of this asymptotics is our main goal.

In our approach, we study asymptotic behavior of eigenvalues and eigenvectors of kinetic matrices along logarithmic straight lines, $\ln k_{i}=\theta_{i} \xi$ in the space of constants. We significantly use the graph representation of chemical reaction networks and demonstrate, that for almost every vector $\left(\theta_{i}\right)$ there exists a simple reaction network which describes the dominant term of this asymptotic. Following the asymptotology terminology (White, 2006), we call this simple network the dominant system. For these dominant system there are explicit formulas for eigenvalues and eigenvectors. The topology of dominant systems is rather simple: they are acyclic networks without branching. This allows us to construct the explicit asymptotics of eigenvectors and eigenvalues. All algorithms are represented topologically by transformation of the graph of reaction (labeled by reaction rate constants). The reaction rate constants for dominant systems may not coincide with constant of original network. In general, they are monomials of the original constants.

This result fully supports the observation by Kruskal (1963): "And the answer quite generally has the form of a new system (well posed problem) for the solution to satisfy, although this is sometimes obscured because the new system is so easily solved that one is led directly to the solution without noticing the intermediate step".

The dominant systems can be used for direct computation of steady states and relaxation dynamics, especially when kinetic information is incomplete, for design of experiments and mining of experimental data, and could serve as a robust first approximation in perturbation theory or for preconditioning. They can be used to answer an important question: given a network model, which are its critical parameters? Many of the parameters of the initial model are no longer present in the dominant system: these parameters are non-critical. Parameters of dominant subsystems indicate putative targets to change the behavior of the large network.

Most of reaction networks are nonlinear, it is nevertheless useful to have an efficient algorithm for solving linear problems. First, nonlinear systems often include linear subsystems, containing reactions that are (pseudo)monomolecular with respect to species internal to the subsystem (at most one internal species is reactant and at most one is product). Second, for binary reactions $A+B \rightarrow \ldots$, if concentrations of species $A$ and $B\left(c_{A}, c_{B}\right)$ are well separated, say $c_{A} \gg c_{B}$ then we can consider this reaction as $B \rightarrow \ldots$ with rate constant proportional to $c_{A}$ which is practically constant, because its relative changes are small in comparison to relative changes of $c_{B}$. We can assume that this condition is satisfied for all but a small fraction of genuinely nonlinear reactions (the set of nonlinear reactions changes in time but remains small). Under such an assumption, nonlinear behavior can be approximated as a sequence of such systems, followed one each other in a sequence of "phase transitions". In these transitions, the order relation between some of species concentrations changes. Some applications of this approach to systems biology are presented by Radulescu et al. (2008). The idea of controllable linearization "by excess" of some reagents is in the background of the efficient experimental technique of temporal analysis of products (TAP), which allows to decipher detailed mechanisms of catalytic reactions (Yablonsky et al., 2003).
In chemical kinetics various fundamental ideas about asymptotical analysis were developed (Klonowski, 1983): quasieqiulibrium asymptotic (QE), quasisteady-state asymptotic (QSS), lumping, and the idea of limiting step.

Most of the works on non-equilibrium thermodynamics deal with the QE approximations and corrections to them, or with applications of these approximations (with or without corrections). There are two basic formulation of the QE approximation: the thermodynamic approach, based on entropy maximum, or the kinetic formulation, based on selection of fast reversible reactions. The very first use of the entropy maximization dates back to the classical work of Gibbs (1902), but it was first claimed for a principle of informational statistical thermodynamics by Jaynes (1963). A very general discussion of the maximum entropy principle with applications to dissipative kinetics is given in the review by Balian et al. (1986). Corrections of QE approximation with applications to physical and chemical kinetics were developed by Gorban et al. (2001) and Gorban and Karlin (2005).

QSS was proposed by Bodenstein (1913) and was elaborated into an important tool for analysis of chemical reaction mechanism and kinetics (Semenov, 1939; Christiansen, 1953; Helfferich, 1989). The classical QSS is based on the relative smallness of concentrations of some of "active" reagents (radicals, substrateenzyme complexes or active components on the catalyst surface) (Aris, 1965; Segel and Slemrod, 1989).

Lumping analysis aims to combine reagents into "quasicomponents" for dimension reduction (Wei and Kuo, 1969; Kuo and Wei, 1969; Li and Rabitz, 1989; Toth et al., 1997).

The concept of limiting step gives the limit simplification: the whole network behaves as a single step. This is the most popular approach for model simplification in chemical kinetics and in many areas beyond kinetics. In the form of a bottleneck approach this approximation is very popular from traffic management to computer programming and communication networks. The proposed asymptotic analysis can be considered as a wide extension of the classical idea of limiting step (Gorban and Radulescu, 2008).

The structure of the paper is as follows. In Section 2 we introduce basic notions and notations. We consider thermodynamic restrictions on the reaction rate constants and demonstrate how appear systems with arbitrary constants (as subsystems of more detailed models). For linear networks, the main theorems which connect ergodic properties with topology of network, are reminded. Four basic ideas of model reduction in chemical kinetics are described: QE, QSS, lumping analysis and limiting steps.

In Section 3, we introduce the dominant system for a simple irreversible catalytic cycle with limiting step. This is just a chain of reactions which appears after deletion the limiting step from the cycle. Even for such simple examples several new observation are presented:

- The relaxation time for a cycle with limiting step is inverse second reaction rate constant.

- For chains of reactions with well separated rate constants left eigenvectors have coordinates close to 0 or 1 , and right eigenvectors have coordinates close to 0 or \pm 1 .

For general reaction networks instead of linear chains appear general acyclic non-branching networks. For them we also provide explicit formulas for eigenvectors and their $0, \pm 1$ asymptotics for well-separated constants (Section 4). In (Section 5) the main algorithm is presented. Section 6 is devoted to a simple demonstration of the algorithm application. In Section 7, we briefly discuss further corrections to dominant systems. The estimates of accuracy are given in Appendix A. 


\section{Main asymptotic ideas in chemical kinetics}

\subsection{Chemical reaction networks}

To define a chemical reaction network, we have to introduce:

- a list of components (species);

- a list of elementary reactions;

- a kinetic law of elementary reactions.

The list of components is just a list of symbols (labels) $A_{1}, \ldots, A_{n}$. Each elementary reaction is represented by its stoichiometric equation

$\sum_{i} \alpha_{s i} A_{i} \rightarrow \sum_{s i} \beta_{s i} A_{i}$

where $s$ enumerates the elementary reaction, and the nonnegative integers $\alpha_{s i}, \beta_{s i}$ are the stoichiometric coefficients. A stoichiometric vector $\gamma_{s}$ with coordinates $\gamma_{s i}=\beta_{s i}-\alpha_{s i}$ is associated with each elementary reaction.

For analysis of closed chemical systems with detailed balance it is usual practice to group reactions in pairs, direct and inverse reactions together, but in more general settings this is not convenient.

A non-negative real extensive variable $N_{i} \geq 0$, amount of $A_{i}$, is associated with each component $A_{i}$. It measures "the number of particles of that species" (in particles, or in moles). The concentration of $A_{i}$ is an intensive variable: $c_{i}=N_{i} / V$, where $V$ is volume. It is necessary to stress, that in many practically important cases the extensive variable $V$ is neither constant, nor the same for all components $A_{i}$. For more details see, for example the book of Yablonskii et al. (1991). For simplicity, we will consider systems with one constant volume and under constant temperature, but it is necessary always keep in mind the possibility to return to general equations. For that conditions, the kinetic equations have the following form

$\frac{\mathrm{d} c}{\mathrm{~d} t}=\sum_{s} w_{s}(c, T) \gamma_{s}+v$,

where $v$ is the vector of external fluxes normalized to unit volume. It may be useful to represent external fluxes as elementary reactions by introduction of new component $\emptyset$ together with incoming and outgoing reactions $\emptyset \rightarrow A_{i}$ and $A_{i} \rightarrow \emptyset$.

The most popular kinetic law of elementary reactions is the mass action law for perfect systems:

$w_{s}(c, T)=k_{s}(T) \prod c_{i}^{\alpha_{s i}}$,

where "kinetic constant" $k_{S}(T)$ depends on temperature $T$. More general kinetic law, which can be used for most of non-ideal (nonperfect) systems is

$w_{s}(c, T)=\varphi_{s} \exp \left(\frac{1}{R T} \sum_{i} \alpha_{s i} \mu_{i}\right)$

where $R$ is the universal gas constant, $\mu_{i}$ is the chemical potential, $\mu_{i}=\partial F(N, T, V) / \partial N_{i}=\partial G(N, T, P) / \partial N_{i}, F$ is the Helmgoltz free energy, $G$ is the Gibbs energy (free enthalpy), $P$ is pressure and $\varphi_{s}>0$ is an intensive variable, kinetic factor, which can depend on any set of intensive variables, first of all, on $T$.

Chemical thermodynamics (Prigogine and Defay, 1954) provides tools of choice for stability analysis of reaction networks (Procaccia and Ross, 1977) and chemical reactors (Aris, 1965). The laws of thermodynamics have been used for analyzing of structural stability of process systems by Hangos et al. (2004). In general reaction network coefficients $k_{s}(3)$ or $\varphi_{s}(4)$ are not independent. In order to respect the second law of thermodynamics, they should satisfy some equations and inequalities. The most famous sufficient condition gives the principle of detailed balance. Let us group the elementary reactions in pairs, direct and inverse reactions, and mark the variables for direct reactions by superscript + , and for inverse reactions by - . Then the principle of detailed balance for general kinetics (4) reads:

$\varphi_{s}^{+}=\varphi_{s}^{-}$

(Feinberg, 1972). For the isothermal mass action law the principle of detailed balance can be formulated as follows: there exists a strictly positive point $c^{*}$ of detailed balance, at this point

$w_{s}^{+}\left(c^{*}\right)=w_{s}^{-}\left(c^{*}\right)$

for all $s$. This is, essentially, the same principle: if we substitute in the general reaction rate (4) the fraction $\mu_{i} / R T$ by $\ln \left(c_{i} / c_{i}^{*}\right)$, then we will get the mass action law, and $\varphi_{s}^{+}=\varphi_{s}^{-}$. The principle of detailed balance is closely related to the microreversibility and Onsager relations.

More general condition was invented by Stueckelberg (1952) for the Boltzmann equation. He produced them from the $S$-matrix unitarity (the quantum complete probability formula). For the general law (4) without direct-inverse reactions grouping for any state the following identity holds:

$\sum_{s} \varphi_{s} \exp \left(\frac{1}{R T} \sum_{i} \alpha_{s i} \mu_{i}\right) \equiv \sum_{s} \varphi_{s} \exp \left(\frac{1}{R T} \sum_{i} \beta_{s i} \mu_{i}\right)$.

Even more general condition which guarantees the second law and has clear microscopic sense (the complete probability does not increase) was obtained by Gorban (1984): for any state

$\sum_{s} \varphi_{s} \exp \left(\frac{1}{R T} \sum_{i} \alpha_{s i} \mu_{i}\right) \geq \sum_{s} \varphi_{s} \exp \left(\frac{1}{R T} \sum_{i} \beta_{s i} \mu_{i}\right)$.

To obtain formulas for the isothermal mass action law, it is sufficient just to apply the general law (4) with constant $\varphi_{s}$ to the perfect free energy $F=R T \sum_{i} c_{i}\left(\ln c_{i}+\mu_{i 0}\right)$ with constant $\mu_{i 0}$. More detailed analysis was presented, by Gorban (1984).

In any case, reaction constants are dependent, and this dependence guarantees stability of equilibrium and existence of global thermodynamic Lyapunov functions for closed systems (2) with $v=0$. Nevertheless, we often study equations for such systems with oscillations, bifurcations, chaos, and other effects, which are impossible in systems with global Lyapunov function. Usually this means that we study a subsystem of a large system, where some of concentrations do not change because they are stabilized by external fluxes or by a large external reservoir. These constant (or very slow) concentrations are included into new reaction constants, and after this redefinition they can loose any thermodynamic property.

\subsection{Linear networks and ergodicity}

In this section, we consider a general network of linear reactions. This network is represented as a directed graph (digraph) (Temkin et al., 1996): vertices correspond to components $A_{i}$, edges correspond to reactions $A_{i} \rightarrow A_{j}$ with kinetic constants $k_{j i}>0$. For each vertex, $A_{i}$, a positive real variable $c_{i}$ (concentration) is defined. A basis vector $e^{i}$ corresponds to $A_{i}$ with components $e_{j}^{i}=\delta_{i j}$, where $\delta_{i j}$ is the Kronecker delta. The kinetic equation for the system is

$\frac{\mathrm{d} c_{i}}{\mathrm{~d} t}=\sum_{j}\left(k_{i j} c_{j}-k_{j i} c_{i}\right)$,

or in vector form: $\dot{c}=K c$. We do not assume any special relation between constants, and consider them as independent quantities. The thermodynamic restrictions on constants are not applicable here because, in general, we study pseudomonomolecular 
systems which are subsystems of larger nonlinear systems and do not represent by themselves closed monomolecular systems.

For any network of linear reactions the matrix of kinetic coefficients $K$ has the following properties:

- non-diagonal elements of $K$ are non-negative;

- diagonal elements of $K$ are non-positive;

- elements in each column of $K$ have zero sum.

For any $K$ with these properties there exists a network of linear reactions with kinetic equation $\dot{c}=K c$. This family of matrices coincide with the family of generators of finite Markov chains, and this class of kinetic equations coincide with the class of inverse Kolmogorov's equations or master equations for the finite Markov chains in continuous time (Meyn and Tweedie, 2009; Meyn, 2007).

A linear conservation law is a linear function defined on the concentrations $b(c)=\sum_{i} b_{i} c_{i}$, whose value is preserved by the dynamics (9). The conservation laws coefficient vectors $b_{i}$ are left eigenvectors of the matrix $K$ corresponding to the zero eigenvalue. The set of all the conservation laws forms the left kernel of the matrix $K$. Eq. (9) always has a linear conservation law: $b^{0}(c)=\sum_{i} c_{i}=$ const. If there is no other independent linear conservation law, then the system is weakly ergodic.

A set $E$ is positively invariant with respect to kinetic Eq. (9), if any solution $c(t)$ that starts in $E$ at time $t_{0}\left(c\left(t_{0}\right) \in E\right)$ belongs to $E$ for $t>t_{0}\left(c(t) \in E\right.$ if $\left.t>t_{0}\right)$. It is straightforward to check that the standard simplex $\Sigma=\left\{c \mid c_{i} \geq 0, \sum_{i} c_{i}=1\right\}$ is positively invariant set for kinetic Eq. (9): just to check that if $c_{i}=0$ for some $i$, and all $c_{j} \geq 0$ then $\dot{c}_{i} \geq 0$. This simple fact immediately implies the following properties of $K$ :

- All eigenvalues $\lambda$ of $K$ have non-positive real parts, $\operatorname{Re} \lambda \leq 0$, because solutions cannot leave $\Sigma$ in positive time.

- If $\operatorname{Re} \lambda=0$ then $\lambda=0$, because intersection of $\Sigma$ with any plane is a polygon, and a polygon cannot be invariant with respect to rotations to sufficiently small angles.

- The Jordan cell of $K$ that corresponds to zero eigenvalue is diagonal-because all solutions should be bounded in $\Sigma$ for positive time.

- The shift in time operator $\exp (K t)$ is a contraction in the $l_{1}$ norm for $t>0$ : there exists such a monotonically decreasing function $\delta(t)(t>0,0<\delta(t)<1, \delta(t) \rightarrow 0$ when $t \rightarrow \infty)$ that for any two solutions of $(9) c(t), c^{\prime}(t) \in \Sigma$

$$
\sum_{i}\left|c_{i}(t)-c_{i}^{\prime}(t)\right| \leq \delta(t) \sum_{i}\left|c_{i}(0)-c_{i}^{\prime}(0)\right| \text {. }
$$

The ergodicity coefficient $\delta(t)$ was introduced by Dobrushin (1956) (see also a book by Seneta, 1981). It can be estimated using the structure of the network graph (Gorban et al., 1986b; Meyn, 2007).

Two vertices are called adjacent if they share a common edge. A path is a sequence of adjacent vertices. A graph is connected if any two of its vertices are linked by a path. A maximal connected subgraph of graph $G$ is called a connected component of $G$. Every graph can be decomposed into connected components.

A directed path is a sequence of adjacent edges where each step goes in direction of an edge. A vertex $A$ is reachable from a vertex $B$, if there exists a directed path from $B$ to $A$.

A non-empty set $V$ of graph vertices forms a sink, if there are no directed edges from $A_{i} \in V$ to any $A_{j} \notin V$. For example, in the reaction graph $A_{1} \leftarrow A_{2} \rightarrow A_{3}$ the one-vertex sets $\left\{A_{1}\right\}$ and $\left\{A_{3}\right\}$ are sinks. A sink is minimal if it does not contain a strictly smaller sink. In the previous example, $\left\{A_{1}\right\},\left\{A_{3}\right\}$ are minimal sinks. Minimal sinks are also called ergodic components.
A digraph is strongly connected, if every vertex $A$ is reachable from any other vertex $B$. Ergodic components are maximal strongly connected subgraphs of the graph, but inverse is not true: there may exist maximal strongly connected subgraphs that have outgoing edges and, therefore, are not sinks.

The weak ergodicity of the network follows from its topological properties.

Theorem 1. The following properties are equivalent (and each one of them can be used as an alternative definition of weak ergodicity):

(i) There exist the only independent linear conservation law for kinetic Eq. (9) (this is $b^{0}(c)=\sum_{i} c_{i}=$ const).

(ii) For any normalized initial state $c(0)\left(b^{0}(c)=1\right)$ there exists a limit state

$c^{*}=\lim _{t \rightarrow \infty} \exp (K t) c(0)$

that is the same for all normalized initial conditions: For all $c$, $\lim _{t \rightarrow \infty} \exp (K t) c=b^{0}(c) c^{*}$.

(iii) For each two vertices $A_{i}, A_{j}(i \neq j)$ we can find such a vertex $A_{k}$ that is reachable both from $A_{i}$ and from $A_{j}$. This means that the following structure exists:

$A_{i} \rightarrow \cdots \rightarrow A_{k} \leftarrow \cdots \leftarrow A_{j}$.

One of the paths can be degenerated: it may be $i=k$ or $j=k$.

(iv) The network has only one minimal sink (one ergodic component).

The proof of this theorem could be extracted from detailed books about Markov chains and networks (Meyn, 2007; Van Mieghem, 2006). In its present form it was published by Gorban et al. (1986b) with explicit estimations of ergodicity coefficients.

For every monomolecular kinetic system, the maximal number of independent linear conservation laws (i.e. the geometric multiplicity of the zero eigenvalue of the matrix $K$ ) is equal to the maximal number of disjoint ergodic components (minimal sinks).

\subsection{Quasiequilibrium ( $Q E)$ or fast equilibrium}

Quasiequilibrium approximation uses the assumption that a group of reactions is much faster than other and goes fast to its equilibrium. We use below superscripts ' $f$ ' and ' $s$ ' to distinguish fast and slow reactions. A small parameter appears in the following form

$\frac{\mathrm{d} c}{\mathrm{~d} t}=\sum_{\sigma, \text { slow }} w_{\sigma}^{\mathrm{s}}(c, T) \gamma_{\sigma}^{s}+\frac{1}{\varepsilon} \sum_{\varsigma, \text { fast }} w_{\varsigma}^{\mathrm{f}}(c, T) \gamma_{\varsigma}^{\mathrm{f}}$.

To separate variables, we have to study the spaces of linear conservation law of the initial system (11) and of the fast subsystem

$\frac{\mathrm{d} c}{\mathrm{~d} t}=\frac{1}{\varepsilon} \sum_{\varsigma, \text { fast }} w_{\varsigma}^{\mathrm{f}}(c, T) \gamma_{\varsigma}^{\mathrm{f}}$.

If they coincide, then the fast subsystem just dominates, and there is no fast-slow separation for variables (all variables are either fast, or constant). But if there exist additional linearly independent linear conservation laws for the fast system, then let us introduce new variables: linear functions $b^{1}(c), \ldots, b^{n}(c)$, where $b^{1}(c), \ldots, b^{m}(c)$ is the basis of the linear conservation laws for the initial system, and $b^{1}(c), \ldots, b^{m+l}(c)$ is the basis of the linear conservation laws for the fast subsystem. Then $b^{m+l+1}(c), \ldots, b^{n}(c)$ are fast variables, $b^{m+1}(c), \ldots, b^{m+l}(c)$ are slow variables, and $b^{1}(c), \ldots, b^{m}(c)$ are constant. The quasiequilibrium manifold is given 
by the equations $\sum_{\varsigma} w_{\varsigma}^{\mathrm{f}}(c, T) \gamma_{\varsigma}^{\mathrm{f}}=0$ and for small $\varepsilon$ it serves as an approximation to a slow manifold. In the old and standard approach it is assumed that system (11) as well as system of fast reactions satisfies the thermodynamic restrictions, and the quasiequilibrium is just a partial thermodynamic equilibrium, and could be defined by conditional extremum of thermodynamic functions. This guarantees global stability of fast subsystems and all the classical singular perturbation theory like Tikhonov theorem could be applied.

Recently, Vora and Daoutidis (2001) took notice that this type of reasoning does not require classical thermodynamic restrictions on constants. For example, let us consider the mass action law kinetics and group the reactions in pairs, direct and inverse reactions. If the set of stoichiometric vectors for fast reactions is linearly independent, then for this system the detailed balance principle holds (obviously), and it demonstrates the "thermodynamic behavior" without connection to classical thermodynamics. This case of "stoichiometrically independent fast reactions" can be generalized for irreversible reactions too (Vora and Daoutidis, 2001). For such fast system the quasiequilbrium manifold has the same nice properties as for thermodynamic partial equilibrium, and approximates slow dynamics for sufficiently small $\varepsilon$.

There are other classes of mass action law subsystems with such a "quasithermodynamic" behavior, which depends on structure, but not on constants. For example, any system of reactions without interactions has such a property (Gorban et al., 1986a). These reactions have the form $\alpha A_{i} \rightarrow \sum \cdots$ : any linear reaction are allowed, as well as reactions like $2 A_{i} \rightarrow A_{j}+A_{k}$, $3 A_{i} \rightarrow A_{j}+A_{k}+A_{l}$, etc. All such fast subsystems can serve for quasiequilibrium approximation, because for them dynamics is globally stable.

Quasiequilibrium manifold approximates exponentially attractive slow manifold and is used in many areas of kinetics either as initial approximation for slow motion, or just by itself (more discussion and further references are presented by Gorban and Karlin, 2005).

\subsection{Quasi-steady state (QSS) or fast species}

The quasisteady-state (or pseudo steady state) assumption was invented in chemistry for description of systems with radicals or catalysts. In the most usual version the species are split in two groups with concentration vectors $c^{s}$ ("slow" or basic components) and $c^{\mathrm{f}}$ ("fast intermediates"). For catalytic reactions there is additional balance for $c^{\mathrm{f}}$, amount of catalyst, usually it is just a sum $b_{\mathrm{f}}=\sum_{i} c_{i}^{\mathrm{f}}$. The amount of the fast intermediates is assumed much smaller than the amount of the basic components, but the reaction rates are of the same order, or even the same (both intermediates and slow components participate in the same reactions). This is the source of a small parameter in the system. Let us scale the concentrations $c^{\mathrm{f}}$ and $c^{\mathrm{s}}$ to the compatible amounts. After that, the fast and slow time appear and we could write $\dot{c}^{\mathrm{s}}=W^{\mathrm{s}}\left(c^{\mathrm{s}}, c^{\mathrm{f}}\right), \dot{c}^{\mathrm{f}}=(1 / \varepsilon) W^{\mathrm{f}}\left(c^{\mathrm{s}}, c^{\mathrm{f}}\right)$, where $\varepsilon$ is small parameter, and functions $W^{s}, W^{f}$ are bounded and have bounded derivatives (are "of the same order"). We can apply the standard singular perturbation techniques. If dynamics of fast components under given values of slow concentrations is stable, then the slow attractive manifold exists, and its zero approximation is given by the system of equations $W^{\mathrm{f}}\left(c^{\mathrm{s}}, c^{\mathrm{f}}\right)=0$. Bifurcations in fast system correspond to critical effects, including ignition and explosion.

This scheme was analyzed many times with plenty of details, examples, and some complications. Exhaustive case study of the simplest enzyme reaction was provided by Segel and Slemrod (1989). For heterogenous catalytic reactions, the book by
Yablonskii et al. (1991) gives analysis of scaling of fast intermediates (there are many kinds of possible scaling). In the context of the Computational Singular Perturbation (CSP) approach, Lam (1993) and Lam and Goussis (1994) developed concept of the CSP radicals. Gorban and Karlin $(2003,2005)$ considered QSS as initial approximation for slow invariant manifold. Analysis of the error of the QSS was provided by Turanyi et al. (1993).

The QE approximation is also extremely popular and useful. It has simpler dynamical properties (respects thermodynamics, for example, and gives no critical effects in fast subsystems of closed systems). Nevertheless, neither radicals in combustion, nor intermediates in catalytic kinetics are, in general, close to quasiequilibrium. They are just present in much smaller amount, and when this amount grows, then the QSS approximation fails.

The simplest demonstration of these two approximation gives the simple reaction: $S+E \leftrightarrow S E \rightarrow P+E$ with reaction rate constants $k_{1}^{ \pm}$and $k_{2}$. The only possible quasiequilibrium appears when the first equilibrium is fast: $k_{1}^{ \pm}=\kappa^{ \pm} / \varepsilon$. The corresponding slow variable is $C^{S}=c_{S}+c_{S E}, b_{E}=c_{E}+c_{S E}=$ const. For the $\mathrm{QE}$ manifold we get a quadratic equation $k_{1}^{-} / k_{1}^{+} c_{S E}=c_{S} c_{E}=\left(C^{S}-c_{S E}\right)\left(b_{E}-c_{S E}\right)$. This equation gives the explicit dependence $c_{S E}\left(C^{S}\right)$, and the slow equation reads $\dot{C}^{s}=-k_{2} C_{S E}\left(C^{s}\right), C^{s}+c_{P}=b_{S}=$ const.

For the QSS approximation of this reaction kinetics, under assumption $b_{E} \ll b_{S}$, we have fast intermediates $E$ and $S E$. For the QSS manifold there is a linear equation $k_{1}^{+} c_{S} c_{E}-k_{1}^{-} c_{S E}-k_{2} c_{S E}=0$, which gives us the explicit expression for $c_{S E}\left(c_{S}\right): c_{S E}=$ $k_{1}^{+} c_{S} b_{E} /\left(k_{1}^{+} c_{S}+k_{1}^{-}+k_{2}\right)$ (the standard Michaelis-Menten formula). The slow kinetics reads $\dot{c}_{S}=-k_{1}^{+} c_{S}\left(b_{E}-c_{S E}\left(c_{S}\right)\right)+k_{1}^{-} c_{S E}\left(c_{S}\right)$. The difference between the QSS and the QE in this example is obvious.

The terminology is not rigorous, and often QSS is used for all singular perturbed systems, and $\mathrm{QE}$ is applied only for the thermodynamic exclusion of fast variables by the maximum entropy (or minimum of free energy, or extremum of another relevant thermodynamic function) principle (MaxEnt). This terminological convention may be convenient. Nevertheless, without any relation to terminology, the difference between these two types of introduction of a small parameter is huge. There exists plenty of generalizations of these approaches, which aim to construct a slow and (almost) invariant manifold, and to approximate fast motion as well. The following references can give a first impression about these methods: Method of Invariant Manifolds (MIM) (Roussel and Fraser, 1991; Gorban and Karlin, 2005), Method of Invariant Grids (MIG), a discrete analogue of invariant manifolds (Gorban et al., 2004), Computational Singular Perturbations (CSP) (Lam, 1993; Lam and Goussis, 1994; Zagaris et al., 2004) Intrinsic Low-Dimensional Manifolds (ILDM) by Maas and Pope (1992), developed further in series of works by Bykov et al. (2006), methods based on the Lyapunov auxiliary theorem (Kazantzis and Kravaris, 2006).

\subsection{Lumping analysis}

Wei and Prater (1962) demonstrated that for (pseudo)monomolecular systems there exist linear combinations of concentrations which evolve in time independently. These linear combinations (quasicomponents) correspond to the left eigenvectors of kinetic matrix: if $l K=\lambda l$ then $\mathrm{d}(l, c) / \mathrm{d} t=(l, c) \lambda$, where the standard inner product $(l, c)$ is concentration of a quasicomponent. They also demonstrated how to find these quasicomponents in a properly organized experiment.

This observation gave rise to a question: how to lump components into proper quasicomponents to guarantee the autonomous dynamics of the quasicomponents with appropriate accuracy. Wei and Kuo studied conditions for exact (Wei and Kuo, 1969) and approximate (Kuo and Wei, 1969) lumping in 
monomolecular and pseudomonomolecular systems. They demonstrated that under certain conditions large monomolecular system could be well-modeled by lower-order system.

More recently, sensitivity analysis and Lie group approach were applied to lumping analysis (Li and Rabitz, 1989; Toth et al., 1997), and more general nonlinear forms of lumped concentrations are used (for example, concentration of quasicomponents could be rational function of $c$ ).

Hutchinson and Luss (1970) studied lumping-analysis of mixtures with many parallel first order reactions. Farkas (1999) generalized these results and characterized those lumping schemes which preserve the kinetic structure of the original system. Coxson and Bischoff (1987) placed lumping analysis in the linear systems theory and demonstrated the relationships between lumpability and the concepts of observability, controllability and minimal realization. Djouad and Sportisse (2002) considered the lumping procedures as efficient techniques leading to non-stiff systems and demonstrated efficiency of developed algorithm on kinetic models of atmospheric chemistry. Lin et al. (2008) formulated an optimal lumping problem as a mixed integer nonlinear programming (MINLP) and demonstrated that it can be efficiently solved with a stochastic optimization method, Tabu Search (TS) algorithm.

The power of lumping using a time-scale based approach was demonstrated by Liao and Lightfoot (1988) and Whitehouse et al. (2004). This computationally cheap approach combines ideas of sensitivity analysis with simple and useful grouping of species with similar lifetimes and similar topological properties caused by connections of the species in the reaction networks. The lumped concentrations in this approach are simply sums of concentrations in groups. For example, species with similar composition and functionalities could be lumped into one single representative species (Pepiot-Desjardins and Pitsch, 2008).

Lumping analysis based both on mathematical arguments and fundamental physical and chemical properties of the components is now one of the main tools for model reduction in highly multicomponent systems, such as the hydrocarbon mixture in petroleum chemistry (Zavala et al., 2004) or biochemical networks in systems biology (Maria, 2006). The optimal solution of lumping problem often requires the exhaustive search, and instead of them various heuristics are used to avoid combinatorial explosion. For the lumping analysis of the systems biology models Dokoumetzidis and Aarons (2009) developed a heuristic greedy search strategy which allowed them to avoid the exhaustive search of proper lumped components.

Procedures of lumping analysis form a part of general algebra of model building and model simplification transformations. Hangos and Cameron (2001) applied formal methods of computer science and artificial intelligence for analysis of this algebra. In particular, a formal method for defining syntax and semantics of process models has been proposed.

The modern systems and control theory provides efficient tools for lumping-analysis. The so-called balanced model reduction was invented in late 1970s (Moore, 1981). For a linear system a set of "target variables" is selected. The dimension of the system $n$ is large, while the number of the target variables, for example, inputs $m$ and outputs $p$, usually satisfies $m, p \ll n$. The balanced model reduction problem can be stated as follows (Gugercin and Antoulas, 2004): find a reduced order system such that the following properties are satisfied:

(i) The approximation error in the target variables is small, and there exists a global error bound.

(ii) System properties, like stability and passivity, are preserved. (iii) The procedure is computationally efficient.
In large dimensions, special efforts are needed to resolve the accuracy/efficiency dilemma and to find efficiently the approximate solution of the model reduction problem (Antoulas and Sorensen, 2002).

Various methods for balanced truncation are developed: Lyapunov balancing, stochastic balancing, bounded real balancing, positive real balancing, and frequency weighted balancing (Gugercin and Antoulas, 2004). Nonlinear generalizations are proposed as well (Lall et al., 2002; Condon and Ivanov, 2004).

\subsection{Limiting steps}

In the IUPAC Compendium of Chemical Terminology (2007) one can find a definition of limiting steps. Rate-controlling (2007): "A rate-controlling (rate-determining or rate-limiting) step in a reaction occurring by a composite reaction sequence is an elementary reaction the rate constant for which exerts a strong effect-stronger than that of any other rate constant-on the overall rate."

Let us complement this definition by additional comment: usually when people are talking about limiting step they expect significantly more: there exists a rate constant which exerts such a strong effect on the overall rate that the effect of all other rate constants together is significantly smaller. For the IUPAC Compendium definition a rate-controlling step always exists, because among the control functions generically exists the biggest one. On the contrary, for the notion of limiting step that is used in practice, there exists a difference between systems with limiting step and systems without limiting step.

During XX century, the concept of the limiting step was revised several times. First simple idea of a "narrow place" (the least conductive step) could be applied without adaptation only to a simple cycle or a chain of irreversible steps that are of the first order (see Chapter 16 of the book Johnston, 1966 or the paper by Boyd, 1978). When researchers try to apply this idea in more general situations they meet various difficulties such as:

- Some reactions have to be "pseudomonomolecular." Their constants depend on concentrations of outer components, and are constant only under condition that these outer components are present in constant concentrations, or change sufficiently slow (i.e. are present in significantly bigger amount).

- Even under fixed or slow outer components concentration, the simple "narrow place" behavior could be spoiled by branching or by reverse reactions. The simplest example is given by the cycle: $A_{1} \leftrightarrow A_{2} \rightarrow A_{3} \rightarrow A_{1}$. Even if the constant of the last step $A_{3} \rightarrow A_{1}$ is the smallest one, the stationary rate may be much smaller than $k_{3} b$ (where $b$ is the overall balance of concentrations, $\left.b=c_{1}+c_{2}+c_{3}\right)$, if the constant of the reverse reaction $A_{2} \rightarrow A_{1}$ is sufficiently big.

In a series of papers, Northrop (1981, 2001) clearly explained these difficulties and suggested that the concept of rate-limiting step is "outmoded". Nevertheless, the main idea of limiting is so attractive that Northrop's arguments stimulated the search for modification and improvement of the main concept.

Ray (1983) proposed the use of sensitivity analysis. He considered cycles of reversible reactions and suggested a definition: The rate-limiting step in a reaction sequence is that forward step for which a change of its rate constant produces the largest effect on the overall rate.

Ray's approach was revised by Brown and Cooper (1993) from the system control analysis point of view (see the book of CornishBowden and Cardenas, 1990). They stress again that there is no unique rate-limiting step specific for an enzyme, and this step, 
even if it exists, depends on substrate, product and effector concentrations.

Near critical conditions the critical simplification appears, which is also a type of limitation, because some reactions become critically important (Yablonsky et al., 2003)

Two classical examples of limiting steps demonstrate us the chain of linear reaction and the linear catalytic cycle, when they include a reaction which is significantly slower, than other reactions.

A linear chain of reactions, $A_{1} \rightarrow A_{2} \rightarrow \cdots A_{n}$, with reaction rate constants $k_{i}$ (for $A_{i} \rightarrow A_{i+1}$ ), gives the first example of limiting steps. Let the reaction rate constant $k_{q}$ be the smallest one. Then we expect the following behavior of the reaction chain in time scale $\gtrsim 1 / k_{q}$ : all the components $A_{1}, \ldots, A_{q-1}$ transform fast into $A_{q}$, and all the components $A_{q+1}, \ldots, A_{n-1}$ transform fast into $A_{n}$, only two components, $A_{q}$ and $A_{n}$ are present (concentrations of other components are small), and the whole dynamics in this time scale can be represented by a single reaction $A_{q} \rightarrow A_{n}$ with reaction rate constant $k_{q}$. This picture becomes more exact when $k_{q}$ becomes smaller with respect to other constants.

The catalytic cycle is one of the most important substructures that we study in reaction networks. In the reduced form the catalytic cycle is a set of linear reactions:

$A_{1} \rightarrow A_{2} \rightarrow \cdots A_{n} \rightarrow A_{1}$.

Reduced form means that in reality some of these reaction are not monomolecular and include some other components (not from the list $\left.A_{1}, \ldots, A_{n}\right)$. But in the study of the isolated cycle dynamics, concentrations of these components are taken as constant and are included into kinetic constants of the cycle linear reactions.

For the constant of elementary reaction $A_{i} \rightarrow$ we use the simplified notation $k_{i}$ because the product of this elementary reaction is known, it is $A_{i+1}$ for $i<n$ and $A_{1}$ for $i=n$. The elementary reaction rate is $w_{i}=k_{i} c_{i}$, where $c_{i}$ is the concentration of $A_{i}$. The kinetic equation is:

$\dot{c}_{i}=k_{i-1} c_{i-1}-k_{i} c_{i}$,

where by definition $c_{0}=c_{n}, k_{0}=k_{n}$, and $w_{0}=w_{n}$. In the stationary state $\left(\dot{c}_{i}=0\right)$, all the $w_{i}$ are equal: $w_{i}=w$. This common rate $w$ we call the cycle stationary rate, and

$w=\frac{b}{\frac{1}{k_{1}}+\ldots \frac{1}{k_{n}}}, \quad c_{i}=\frac{w}{k_{i}}$,

where $b=\sum_{i} c_{i}$ is the conserved quantity for reactions in constant volume. Let one of the constants, $k_{\min }$, be much smaller than others (let it be $k_{\min }=k_{n}$ ):

$k_{i} \gg k_{\min } \quad$ if $i \neq n$.

In this case, in linear approximation $w=k_{n} b$,

$c_{n}=b\left(1-\sum_{i<n} \frac{k_{n}}{k_{i}}\right) \quad$ and $\quad c_{i}=b \frac{k_{n}}{k_{i}}$ for $i \neq n$.

The simplest zero order approximation for the steady state gives

$c_{n}=b, \quad c_{i}=0(i \neq n)$

This is trivial: all the concentration is collected at the starting point of the "narrow place," but may be useful as an origin point for various approximation procedures.

So, the stationary rate of a cycle is determined by the smallest constant, $k_{\min }$, if it is much smaller than the constants of all other reactions (14):

$w \approx k_{\min } b$.

In that case we say that the cycle has a limiting step with constant $k_{\min }$.

\section{Dynamics of catalytic cycle with limiting step}

\subsection{Eigenvalues}

There is significant difference between the examples of limiting steps for the chain of reactions and for irreversible cycle. For the chain, the steady state does not depend on non-zero rate constants. It is just $c_{n}=b, c_{1}=c_{2}=\cdots=c_{n-1}=0$. The smallest rate constant $k_{q}$ gives the smallest positive eigenvalue, the relaxation time is $\tau=1 / k_{q}$. The corresponding approximation of eigenmode (right eigenvector) $r^{1}$ has coordinates: $r_{1}^{1}=\cdots=r_{q-1}^{1}$ $=0, r_{q}^{1}=1, r_{q+1}^{1}=\cdots=r_{n-1}^{1}=0, r_{n}=-1$. This exactly corresponds to the statement that the whole dynamics in the time scale $\gtrsim 1 / k_{q}$ can be represented by a single reaction $A_{q} \rightarrow A_{n}$ with reaction rate constant $k_{q}$. The left eigenvector for eigenvalue $k_{q}$ has approximation $l^{1}$ with coordinates $l_{1}^{1}=l_{2}^{1}=\cdots=l_{q}^{1}=1, l_{q+1}^{1}=$ $\cdots=l_{n}^{1}=0$. This vector provides the almost exact lumping on time scale $\gtrsim 1 / k_{q}$. Let us introduce a new variable $c_{\text {lump }}=\sum_{i} l_{i} c_{i}$, i.e. $c_{\text {lump }}=c_{1}+c_{2}+\cdots+c_{q}$. For the time scale $\gtrsim 1 / k_{q}$ we can write $c_{\text {lump }}+c_{n} \approx b, \mathrm{~d} c_{\text {lump }} / \mathrm{d} t \approx-k_{q} c_{\text {lump }}, \mathrm{d} c_{n} / \mathrm{d} t \approx k_{q} c_{\text {lump }}$.

In the example of a cycle, we approximate the steady state, that is, the right eigenvector $r^{0}$ for zero eigenvalue (the left eigenvector is known and corresponds to the main linear balance $\left.b: l_{i}^{0} \equiv 1\right)$. In the zero-order approximation, this eigenvector has coordinates $r_{1}^{0}=\cdots=r_{n-1}^{0}=0, r_{n}^{0}=1$.

If $k_{n} / k_{i}$ is small for all $i<n$, then the kinetic behavior of the cycle is determined by a linear chain of $n-1$ reactions $A_{1} \rightarrow A_{2} \rightarrow \cdots A_{n}$, which we obtain after cutting the limiting step. The characteristic equation for an irreversible cycle, $\prod_{i=1}^{n}\left(\lambda+k_{i}\right)-\prod_{i=1}^{n} k_{i}=0$, tends to the characteristic equation for the linear chain, $\lambda \prod_{i=1}^{n-1}\left(\lambda+k_{i}\right)=0$, when $k_{n} \rightarrow 0$.

The characteristic equation for a cycle with limiting step $\left(k_{n} / k_{i} \ll 1\right)$ has one simple zero eigenvalue that corresponds to the conservation law $\sum c_{i}=b$ and $n-1$ non-zero eigenvalues

$\lambda_{i}=-k_{i}+\delta_{i}(i<n)$.

where $\delta_{i} \rightarrow 0$ when $\sum_{i<n} k_{n} / k_{i} \rightarrow 0$.

A cycle with limiting step (12) has real eigenspectrum and demonstrates monotonic relaxation without damped oscillations. Of course, without limitation such oscillations could exist, for example, when all $k_{i} \equiv k>0,(i=1, \ldots, n)$.

The relaxation time of a stable linear system (12) is, by definition, $\tau=1 / \min \left\{\operatorname{Re}\left(-\lambda_{i}\right)\right\}(\lambda \neq 0)$. For small $k_{n}, \tau \approx 1 / k_{\tau}, k_{\tau}=$ $\min \left\{k_{i}\right\},(i=1, \ldots, n-1)$. In other words, for a cycle with limiting step, $k_{\tau}$ is the second slowest rate constant: $k_{\min } \ll k_{\tau} \leq \cdots$.

\subsection{Eigenvectors for reaction chain and for catalytic cycle with limiting step}

Let the irreversible cycle include a limiting step: $k_{n} \ll k_{i}$ $(i=1, \ldots, n-1)$ and, in addition, $k_{n} \ll\left|k_{i}-k_{j}\right|(i, j=1, \ldots, n-1$, $i \neq j$ ), then the eigenvectors of the kinetic matrix almost coincide with the eigenvectors for the linear chain of reactions $A_{1} \rightarrow A_{2} \rightarrow \cdots A_{n}$, with reaction rate constants $k_{i}$ (for $A_{i} \rightarrow A_{i+1}$ ) (Gorban and Radulescu, 2008).

The kinetic equation for the linear chain is

$\dot{c}_{i}=k_{i-1} c_{i-1}-k_{i} c_{i}$

The coefficient matrix $K$ of these equations is very simple. It has non-zero elements only on the main diagonal, and one position below. The eigenvalues of $K$ are $-k_{i}(i=1, \ldots, n-1)$ and 0 . The left and right eigenvectors for 0 eigenvalue, $l^{0}$ and $r^{0}$, are

$l^{0}=(1,1, \ldots, 1), \quad r^{0}=(0,0, \ldots, 0,1)$,

all coordinates of $l^{0}$ are equal to 1 , the only non-zero coordinate of $r^{0}$ is $r_{n}^{0}$ and we represent vector-column $r^{0}$ in row. 
Below we use explicit form of $K$ left and right eigenvectors. Let vector-column $r^{i}$ and vector-row $l^{i}$ be right and left eigenvectors of $K$ for eigenvalue $-k_{i}$. For coordinates of these eigenvectors we use notation $r_{j}^{i}$ and $l_{j}^{i}$. Let us choose a normalization condition $r_{i}^{i}=l_{i}^{i}=1$. It is straightforward to check that $r_{j}^{i}=0(j<i)$ and $l_{j}^{i}=0 \quad(j>i)$, $r_{j+1}^{i}=k_{j} r_{j} /\left(k_{j+1}-k_{i}\right)(j \geq i)$ and $l_{j-1}^{i}=k_{j-1} l_{j} /\left(k_{j-1}-k_{j}\right)(j \leq i)$, and

$r_{i+m}^{i}=\prod_{j=1}^{m} \frac{k_{i+j-1}}{k_{i+j}-k_{i}}, \quad l_{i-m}^{i}=\prod_{j=1}^{m} \frac{k_{i-j}}{k_{i-j}-k_{i}}$.

It is convenient to introduce formally $k_{0}=0$. Under selected normalization condition, the inner product of eigenvectors is: $l^{i} r^{j}=\delta_{i j}$, where $\delta_{i j}$ is the Kronecker delta.

If the rate constants any two constants, $k_{i}, k_{j}$ are connected by relation $k_{i} \gg k_{j}$ or $k_{i} \ll k_{j}$ (i.e. they are well separated), then

$\frac{k_{i-j}}{k_{i-j}-k_{i}} \approx \begin{cases}1 & \text { if } k_{i} \ll k_{i-j} ; \\ 0 & \text { if } k_{i} \gg k_{i-j} .\end{cases}$

Hence, $\left|l_{i-m}^{i}\right| \approx 1$ or $\left|l_{i-m}^{i}\right| \approx 0$. To demonstrate that also $\left|r_{i+m}^{i}\right| \approx 1$ or $\left|r_{i+m}^{i}\right| \approx 0$, we shift nominators in the product (21) on such a way:

$r_{i+m}^{i}=\frac{k_{i}}{k_{i+m}-k_{i}} \prod_{j=1}^{m-1} \frac{k_{i+j}}{k_{i+j}-k_{i}}$.

Exactly as in (22), each multiplier $k_{i+j} /\left(k_{i+j}-k_{i}\right)$ here is either almost 1 or almost 0 , and $k_{i} /\left(k_{i+m}-k_{i}\right)$ is either almost 0 or almost -1 . In this zero-one asymptotics

$l_{i}^{i}=1, l_{i-m}^{i} \approx 1$

if $k_{i-j}>k_{i}$ for all $j=1, \ldots, m$ else $l_{i-m}^{i} \approx 0$;

$r_{i}^{i}=1, r_{i+m}^{i} \approx-1$

if $k_{i+j}>k_{i}$ for all $j=1, \ldots m-1$

and $k_{i+m}<k_{i}$ else $r_{i+m}^{i} \approx 0$.

In this asymptotic (Fig. 1), only two coordinates of right eigenvector $r^{i}$ can have non-zero values, $r_{i}^{i}=1$ and $r_{i+m}^{i} \approx-1$ where $m$ is the first such positive integer that $i+m<n$ and $k_{i+m}<k_{i}$. Such $m$ always exists because $k_{n}=0$. For left eigenvector $l^{i}, l_{i}^{i} \approx \ldots l_{i-q}^{i} \approx 1$ and $l_{i-q-j}^{i} \approx 0$ where $j>0$ and $q$ is the first such positive integer that $i-q-1>0$ and $k_{i-q-1}<k_{i}$. It is possible that such $q$ does not exist. In that case, all $l_{i-j}^{i} \approx 1$ for $j \geq 0$. It is straightforward to check that in this asymptotic $l^{i} r^{j}=\delta_{i j}$.

The simplest example gives the order $k_{1} \gg k_{2} \gg \cdots \gg k_{n-1}$ : $l_{i-j}^{i} \approx 1$ for $j \geq 0, r_{i}^{i}=1, r_{i+1}^{i} \approx-1$ and all other coordinates of eigenvectors are close to zero. For the inverse order, $k_{1} \ll k_{2} \ll \cdots \ll k_{n-1}, l_{i}^{i}=1, r_{i}^{i}=1, r_{n}^{i} \approx-1$ and all other coordinates of eigenvectors are close to zero.

For less trivial example, let us find the asymptotic of left and right eigenvectors for a chain of reactions:

$A_{1} \stackrel{5}{\rightarrow} A_{2} \stackrel{3}{\rightarrow} A_{3} \stackrel{4}{\rightarrow} A_{4} \stackrel{1}{\rightarrow} A_{5} \stackrel{2}{\rightarrow} A_{6}$,

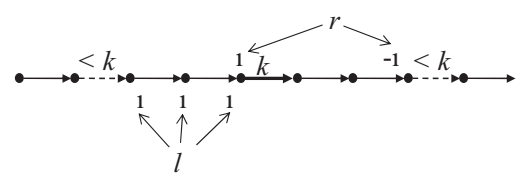

Fig. 1. Graphical representation of eigenvectors approximation for the linear chain of reactions with well separated constants. To find the left $(l)$ and right $(r)$ eigenvectors for eigenvalue $k$ it is necessary to delete from the chain all the reactions with the rate constants $<k$ (dashed lines) and to find the maximal connected interval, where the reaction with constant $k$ (bold arrow) is situated. The right eigenvector $r$ has coordinate 1 for the vertex, which is the beginning of the reaction with constant $k$, and coordinate -1 for the vertex, which is end of the interval in the direction of reactions. The left eigenvector $l$ has coordinate 1 for the beginning of the reaction with constant $k$ and for all preceding vertices from the connected interval. All other coordinates of $r$ and $l$ are zero. where the upper index marks the order of rate constants: $k_{4} \gg k_{5} \gg k_{2} \gg k_{3} \gg k_{1}\left(k_{i}\right.$ is the rate constant of reaction $\left.A_{i} \rightarrow \cdots\right)$. tics:

For left eigenvectors, rows $l^{i}$, we have the following asympto-

$l^{1} \approx(1,0,0,0,0,0), \quad l^{2} \approx(0,1,0,0,0,0)$,

$l^{3} \approx(0,1,1,0,0,0), \quad l^{4} \approx(0,0,0,1,0,0)$,

$l^{5} \approx(0,0,0,1,1,0)$.

For right eigenvectors, columns $r^{i}$, we have the following asymptotics (we write vector-columns in rows):

$r^{1} \approx(1,0,0,0,0,-1), \quad r^{2} \approx(0,1,-1,0,0,0)$,

$r^{3} \approx(0,0,1,0,0,-1), \quad r^{4} \approx(0,0,0,1,-1,0)$,

$r^{5} \approx(0,0,0,0,1,-1)$.

The corresponding approximation to the general solution of the kinetic equations is

$c(t)=\left(l^{0}, c(0)\right) r^{0}+\sum_{i=1}^{n-1}\left(l^{i} c(0)\right) r^{i} \exp \left(-k_{i} t\right)$,

where $c(0)$ is the initial concentration vector, and for left and right eigenvectors $l^{i}$ and $r^{i}$ we use their zero-one asymptotic. In other words, approximation of the left eigenvectors provides us with almost exact lumping (for analysis of exact lumping see the paper by Li and Rabitz, 1989).

\section{Acyclic non-branching network: explicit formulas for eigenvectors}

So, to analyze asymptotic of eigenvalues and eigenvectors for a irreversible cycle, we cut the reaction with the smallest constant, get a linear chain, and analyze the eigenvalues and eigenvectors for this chain. For a general multiscale reaction network (instead of a cycle) we will come, after some surgery, to acyclic nonbranching reaction networks (instead of a linear chain).

For any network without branching, we can simplify the notation for the kinetic constants, by introducing $\kappa_{i}=k_{j i}$ for the only reaction $A_{i} \rightarrow A_{j}$, or $\kappa_{i}=0$, if there is no such a reaction. Also it is useful to introduce a map $\phi$ on the set of vertices: $\phi(i)=j$, if there exist reaction $A_{i} \rightarrow A_{j}$, and $\phi(i)=i$ if there are no outgoing reactions from the $A_{i} \rightarrow A_{j}$. For iterations of the map $\phi$ we use notation $\phi^{q}$.

For an acyclic non-branching reaction network, for any vertex $A_{i}$ there is an eigenvalue $-\kappa_{i}$ and the corresponding eigenvector. If $A_{i}$ is a sink vertex, then this eigenvalue is zero. For left and right eigenvectors of $K$ that correspond to $A_{i}$ we use notations $l^{i}$ (vectorrow) and $r^{i}$ (vector-column), correspondingly.

Let us suppose that $A_{f}$ is a sink vertex of the network. Its associated right and left eigenvectors corresponding to the zero eigenvalue are given by: $r_{j}^{i}=\delta_{i j} ; l_{j}^{i}=1$ if and only if $\phi^{q}(j)=i$ for some $q>0$.

For non-zero eigenvalues, right eigenvectors will be constructed by recurrence starting from the vertex $A_{i}$ and moving in the direction of the flow. The construction is in opposite direction for left eigenvectors.

For right eigenvector $r^{i}$ only coordinates $r_{\phi^{k}(i)}^{i}\left(k=0,1, \ldots, \tau_{i}\right)$ could have non-zero values, and

$$
\begin{aligned}
r_{\phi^{k+1}(i)}^{i} & =\frac{\kappa_{\phi^{k}(i)}}{\kappa_{\phi^{k+1}(i)}-\kappa_{i}} r_{\phi^{k}(i)}^{i}=\prod_{j=0}^{k} \frac{\kappa_{\phi^{j}(i)}}{\kappa_{\phi^{j+1}(i)}-\kappa_{i}} \\
& =\frac{\kappa_{i}}{\kappa_{\phi^{k+1}(i)}-\kappa_{i}} \prod_{j=0}^{k-1} \frac{\kappa_{\phi^{j+1}(i)}}{\kappa_{\phi^{j+1}(i)}-\kappa_{i}} .
\end{aligned}
$$

For left eigenvector $l^{i}$ coordinate $l_{j}^{i}$ could have non-zero value only if there exists such $q \geq 0$ that $\phi^{q}(j)=i$ (this $q$ is unique because the 


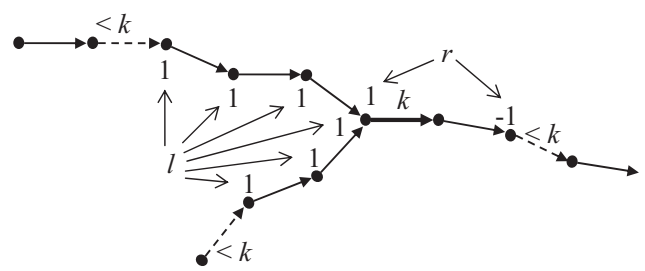

Fig. 2. Graphical representation of eigenvectors approximation for the acyclic nonbranching reaction network with well separated constants (compare to Fig. 1). The eigenvalue $-k$ corresponds to the reaction $A_{i} \rightarrow A_{\phi(i)}$ (bold arrow). To the right from $A_{i}$ are vertices $A_{\phi^{q}(i)}$ and to the left are those $A_{j}$, for which there exists such $q$ that $\phi^{q}(j)=i$. The reactions with the rate constants $<k$ (dashed lines) are deleted from the network. The right and left eigenvectors could have non-zero coordinates only for vertices from the maximal connected subgraph of the presented graph, where the $A_{i}$ is situated. The right eigenvector $r$ has coordinate 1 for $A_{i}$ (beginning of the bold arrow), and coordinate -1 for the vertex, which is the minimal in that connected subgraph. The left eigenvector $l$ has coordinate 1 for the beginning of the reaction with constant $k$ and for all preceding vertices from the subgraph. All other coordinates of $r$ and $l$ are zero.

system is acyclic):

$l_{j}^{i}=\frac{\kappa_{j}}{\kappa_{j}-\kappa_{i}} l_{\phi(j)}^{i}=\prod_{k=0}^{q-1} \frac{\kappa_{\phi^{k}(j)}}{\kappa_{\phi^{k}(j)}-\kappa_{i}}$

For well separated constants, we can write the asymptotic representation explicitly, analogously to (23) (Fig. 2). For left eigenvectors, $l_{i}^{i}=1$ and $l_{j}^{i}=1$ (for $i \neq j$ ) if there exists such $q$ that $\phi^{q}(j)=i$, and $\kappa_{\phi^{d}(j)}>\kappa_{i}$ for all $d=0, \ldots, q-1$, else $l_{j}^{i}=0$. For right eigenvectors, $r_{i}^{i}=1$ and $r_{\phi^{k}(i)}^{i}=-1$ if $\kappa_{\phi^{k}(i)}<\kappa_{i}$ and for all positive $m<k$ inequality $\kappa_{\phi^{m}(i)}>\phi_{i} \kappa_{i}$ holds, i.e. $k$ is first such positive integer that $\kappa_{\phi^{k}(i)}<\kappa_{i}$ (for fixed point $A_{p}$ we use $\kappa_{p}=0$ ). Vector $r^{i}$ has not more than two non-zero coordinates. It is straightforward to check that in this asymptotic $l^{i} r^{j}=\delta_{i j}$.

For example, let us find that asymptotic for a branched acyclic system of reactions:

$A_{1} \stackrel{7}{\rightarrow} A_{2} \stackrel{5}{\rightarrow} A_{3} \stackrel{6}{\rightarrow} A_{4} \stackrel{2}{\rightarrow} A_{5} \stackrel{4}{\rightarrow} A_{8}, \quad A_{6} \stackrel{1}{\rightarrow} A_{7} \stackrel{3}{\rightarrow} A_{4}$.

where the upper index marks the order of rate constants: $\kappa_{6}>\kappa_{4}>\kappa_{7}>\kappa_{5}>\kappa_{2}>\kappa_{3}>\kappa_{1} \quad\left(\kappa_{i}\right.$ is the rate constant of reaction $\left.A_{i} \rightarrow \cdots\right)$.

For zero eigenvalue, the left and right eigenvectors are

$l^{8}=(1,1,1,1,1,1,1,1,1), \quad r^{8}=(0,0,0,0,0,0,0,1)$.

For left eigenvectors, rows $l^{i}$, that correspond to non-zero eigenvalues we have the following asymptotics:

$l^{1} \approx(1,0,0,0,0,0,0,0), \quad l^{2} \approx(0,1,0,0,0,0,0,0)$,

$l^{3} \approx(0,1,1,0,0,0,0,0), \quad l^{4} \approx(0,0,0,1,0,0,0,0)$,

$l^{5} \approx(0,0,0,1,1,1,1,0), \quad l^{6} \approx(0,0,0,0,0,1,0,0)$.

$l^{7} \approx(0,0,0,0,0,1,1,0)$.

For the corresponding right eigenvectors, columns $r^{i}$, we have the following asymptotics (we write vector-columns in rows):

$r^{1} \approx(1,0,0,0,0,0,0,-1), \quad r^{2} \approx(0,1,-1,0,0,0,0,0)$,

$r^{3} \approx(0,0,1,0,0,0,0,-1), \quad r^{4} \approx(0,0,0,1,-1,0,0,0)$,

$r^{5} \approx(0,0,0,0,1,0,0,-1), \quad r^{6} \approx(0,0,0,0,0,1,-1,0)$,

$r^{7} \approx(0,0,0,0,-1,0,1,0)$.

\section{Calculating the dominant system for a linear multiscale network}

\subsection{Problem statement}

We study asymptotical behavior of the transformation of the kinetic matrix $K$ to the normal form along the lines $\ln k_{i j}=\theta_{i j} \xi$ when $\xi \rightarrow \infty$. For almost all direction vectors $\left(\theta_{i j}\right)$ (outside several hyperplanes) there exists a minimal reaction network which reaction rate constants are monomials of $k_{i j}\left(\prod_{i j} k_{i j}^{f_{i j}}\right.$, where $f_{i j}$ are not obligatory positive numbers) and eigenvectors and eigenvalues approximate the eigenvectors and eigenvalues when $\xi \rightarrow \infty$ with arbitrary high relative accuracy. We call this minimal system the dominant system. Existence of dominant systems is proven by direct construction (this Section) and estimates of accuracy of approximations (Appendix).

The dominant systems coincide for vectors $\left(\theta_{i j}\right)$ from some polyhedral cones. Therefore, we do not need to study a given value of $\left(\theta_{i j}\right)$ but rather have to build these cones together with the correspondent dominant systems. The following formal rule ("assumption of well separated constants") allows us to simplify this task: if in construction of dominant systems we need to compare two monomials, $M_{f}=\prod_{i j} k_{i j}^{f_{i j}}$ and $M_{g}=\prod_{i j} k_{i j}^{g_{i j}}$ then we can always state that either $M_{f} \gg M_{g}$ or $M_{f} \ll M_{g}$ and consider the logarithmic hyperplane $M_{f}=M_{g}$ as a boundary between different cones. At the end, we can join all cones with the same dominant system. We are interested in robust asymptotic and do not analyze directions $\left(\theta_{i j}\right)$ which belong to the boundary hyperplanes. This robust asymptotic with well separated constants and acyclic dominant systems is typical because the exclusive direction vectors belon to a finite number of hyperplanes.

There may be other approaches based on: (i) the Maslov dequantization and idempotent algebras (Litvinov and Maslov, 2005), (ii) the limit of log-uniform distributions in wide boxes of constants under some conditions (Feng et al., 2004; Gorban and Radulescu, 2008), or (iii) on consideration of all possible orderings of all monomials with integer exponents and construction of correspondent dominant systems (Robbiano, 1985 proved that there exists only a final number of such orderings and enumerated all of them, see also the book by Greuel and Pfister, 2002). They give the same final result but with different intermediate steps.

\subsection{Auxiliary operations}

\subsubsection{From reaction network to auxiliary dynamical system}

Let us consider a reaction network $\mathcal{W}$ with a given structure and fixed ordering of constants. The set of vertices of $\mathcal{W}$ is $\mathcal{A}$ and the set of elementary reactions is $\mathcal{R}$. Each reaction from $\mathcal{R}$ has the form $A_{i} \rightarrow A_{j}, A_{i}, A_{j} \in \mathcal{A}$. The corresponding constant is $k_{j i}$. For each $A_{i} \in \mathcal{A}$ we define $\kappa_{i}=\max _{j}\left\{k_{j i}\right\}$ and $\phi(i)=\arg \max _{j}\left\{k_{j i}\right\}$. In addition, $\phi(i)=i$ if $k_{j i}=0$ for all $j$.

The auxiliary discrete dynamical system for the reaction network $\mathcal{W}$ is the dynamical system $\Phi=\Phi_{\mathcal{W}}$ defined by the map $\phi$ on the finite set $\mathcal{A}$. The auxiliary reaction network (Fig. 3) $\mathcal{V}=\mathcal{V}_{\mathcal{W}}$ has the same set of vertices $\mathcal{A}$ and the set of reactions $A_{i} \rightarrow A_{\phi(i)}$ with reaction constants $\kappa_{i}$. Auxiliary kinetics is described by $\dot{c}=\tilde{K} c$, where $\tilde{K}_{i j}=-\kappa_{j} \delta_{i j}+\kappa_{j} \delta_{i \phi(j)}$.

\subsubsection{Decomposition of discrete dynamical systems on finite sets}

Discrete dynamical system on a finite set $V=\left\{A_{1}, A_{2}, \ldots, A_{n}\right\}$ is a semigroup $1, \phi, \phi^{2}, \ldots$, where $\phi$ is a map $\phi: V \rightarrow V . A_{i} \in V$ is a periodic point, if $\phi^{l}\left(A_{i}\right)=A_{i}$ for some $l>0$; else $A_{i}$ is a transient point. A cycle of period $l$ is a sequence of $l$ distinct periodic points $A, \phi(A), \phi^{2}(A), \ldots, \phi^{l-1}(A)$ with $\phi^{l}(A)=A$. A cycle of period one consists of one fixed point, $\phi(A)=A$. Two cycles, $C, C^{\prime}$ either coincide or have empty intersection.

The set of periodic points, $V^{\mathrm{p}}$, is always non-empty. It is a union of cycles: $V^{\mathrm{p}}=\cup_{j} C_{j}$. For each point $A \in V$ there exist such a positive integer $\tau(A)$ and a cycle $C(A)=C_{j}$ that $\phi^{q}(A) \in C_{j}$ for $q \geq \tau(A)$. In that case we say that $A$ belongs to basin of attraction of cycle $C_{j}$ and use notation $\operatorname{Att}\left(C_{j}\right)=\left\{A \mid C(A)=C_{j}\right\}$. Of course, $C_{j} \subset \operatorname{Att}\left(C_{j}\right)$. For different 


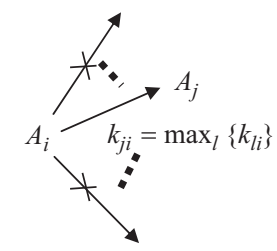

Fig. 3. Construction of the auxiliary reaction network by pruning. For every vertex, it is necessary to leave the outgoing reaction with maximal reaction rate constant. Other reactions should be deleted.

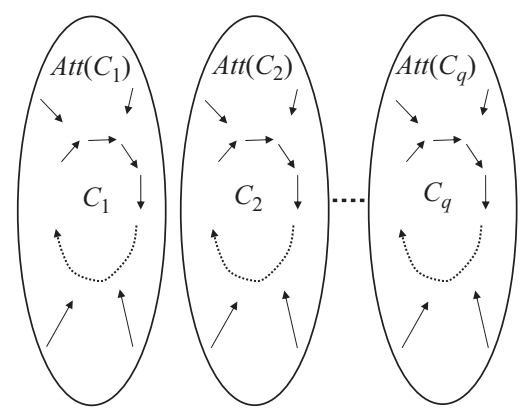

Fig. 4. Decomposition of a discrete dynamical system.

cycles, $\operatorname{Att}\left(C_{j}\right) \cap \operatorname{Att}\left(C_{l}\right)=\emptyset$. If $A$ is periodic point then $\tau(A)=0$. For transient points $\tau(A)>0$.

So, the phase space $V$ is divided onto subsets $\operatorname{Att}\left(C_{j}\right)$ (Fig. 4). Each of these subsets includes one cycle (or a fixed point, that is a cycle of length 1$)$. Sets $\operatorname{Att}\left(C_{j}\right)$ are $\phi$-invariant: $\phi\left(\operatorname{Att}\left(C_{j}\right)\right) \subset \operatorname{Att}\left(C_{j}\right)$. The set $\operatorname{Att}\left(C_{j}\right) \backslash C_{j}$ consist of transient points and there exists such positive integer $\tau$ that $\phi^{q}\left(\operatorname{Att}\left(C_{j}\right)\right)=C_{j}$ if $q \geq \tau$.

Discrete dynamical systems on a finite sets correspond to graphs without branching points. Notice that for the graph that represents a discrete dynamic system, attractors are ergodic components, while basins are connected components.

\subsection{Algorithm for calculating the dominant system}

For this general case, the algorithm consists of two main procedures: (i) cycles gluing and (ii) cycles restoration and cutting.

\subsubsection{Cycles gluing}

Let us start from a reaction network $\mathcal{W}$ with a given structure and fixed ordering of constants. The set of vertices of $\mathcal{W}$ is $\mathcal{A}$ and the set of elementary reactions is $\mathcal{R}$.

If all attractors of the auxiliary dynamic system $\Phi_{\mathcal{W}}$ are fixed points $A_{f 1}, A_{f 2}, \ldots, \in \mathcal{A}$, then the auxiliary reaction network is acyclic, and the auxiliary kinetics approximates relaxation of the whole network $\mathcal{W}$.

In general case, let the system $\Phi_{\mathcal{W}}$ have several attractors that are not fixed points, but cycles $C_{1}, C_{2}, \ldots$ with periods $\tau_{1}, \tau_{2}, \ldots>1$. By gluing these cycles in points, we transform the reaction network $\mathcal{W}$ into $\mathcal{W}^{1}$. The dynamical system $\Phi_{\mathcal{W}}$ is transformed into $\Phi^{1}$. For these new system and network, the connection $\Phi^{1}=\Phi_{\mathcal{W}^{1}}$ persists: $\Phi^{1}$ is the auxiliary discrete dynamical system for $\mathcal{W}^{1}$.

For each cycle, $C_{i}$, we introduce a new vertex $A^{i}$. The new set of vertices, $\mathcal{A}^{1}=\mathcal{A} \cup\left\{A^{1}, A^{2}, \ldots\right\} \backslash\left(\cup_{i} C_{i}\right)$ (we delete cycles $C_{i}$ and add vertices $A^{i}$ ).

All the reaction $A \rightarrow B$ from the initial set $\mathcal{R},(A, B \in \mathcal{A})$ can be separated into 5 groups:

(i) both $A, B \notin \cup_{i} C_{i}$;

(ii) $A \notin \cup_{i} C_{i}$, but $B \in C_{i}$;

(iii) $A \in C_{i}$, but $B \notin \cup_{i} C_{i}$;

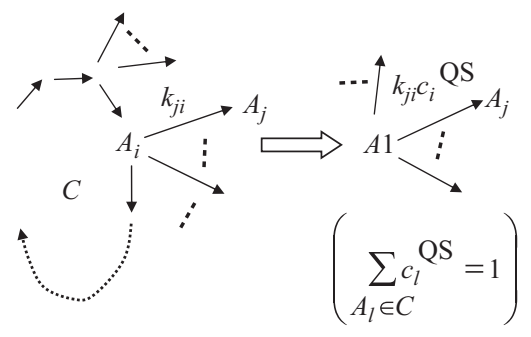

Fig. 5. Gluing a cycle with rate constants renormalization. $c_{l}^{\mathrm{QS}}$ are the quasistationary concentrations on the cycle. After gluing, we have to leave the outgoing from $A^{1}$ reaction with the maximal renormalized rate constant, and delete others.

(iv) $A \in C_{i}, B \in C_{j}, i \neq j$;

(v) $A, B \in C_{i}$.

Reactions from the first group do not change. Reaction from the second group transforms into $A \rightarrow A^{i}$ (to the whole glued cycle) with the same constant. Reaction of the third type changes into $A^{i} \rightarrow B$ with the rate constant renormalization: let the cycle $C^{i}$ be the following sequence of reactions $A_{1} \rightarrow A_{2} \rightarrow \cdots A_{\tau_{i}} \rightarrow A_{1}$, and the reaction rate constant for $A_{i} \rightarrow A_{i+1}$ is $k_{i}\left(k_{\tau_{i}}\right.$ for $\left.A_{\tau_{i}} \rightarrow A_{1}\right)$. For the limiting reaction of the cycle $C_{i}$ we use notation $k_{\lim i}$. If $A=A_{j}$ and $k$ is the rate reaction for $A \rightarrow B$, then the new reaction $A^{i} \rightarrow B$ has the rate constant $k k_{\lim i} / k_{j}$. This corresponds to a quasistationary distribution on the cycle (15). The new rate constant is smaller than the initial one: $k k_{\lim i} / k_{j}<k$, because $k_{\lim i}<k_{j}$ due to definition of limiting constant. The same constant renormalization is necessary for reactions of the fourth type. These reactions transform into $A^{i} \rightarrow A^{j}$. Finally, reactions of the fifth type vanish.

After we glue all the cycles (Fig. 5) of auxiliary dynamical system in the reaction network $\mathcal{W}$, we get $\mathcal{W}^{1}$. Let us assign $\mathcal{W}:=\mathcal{W}^{1}, \mathcal{A}:=\mathcal{A}^{1}$ and iterate until we obtain an acyclic network and exit. This acyclic network is a "forest" and consists of trees oriented from leafs to a root. The number of such trees coincide with the number of fixed points in the final network.

After gluing we can identify the reactions, which will be included into the dominant system. Their constants are the critical parameters of the networks. The list of these parameters, consists of all reaction rates of the final acyclic auxiliary network, and of the rate constants of the glued cycles, but without their limiting steps. Some of these parameters are rate constants of the initial network, other have the monomial structure. Other constants and corresponding reactions do not participate in the following operations. To form the structure of the dominant network, we need one more procedure.

\subsubsection{Cycles restoration and cutting}

We start the reverse process from the glued network $\mathcal{V}^{m}$ on $\mathcal{A}^{m}$. On a step back, from the set $\mathcal{A}^{m}$ to $\mathcal{A}^{m-1}$ and so on, some of glued cycles should be restored and cut. On the $q$ th step we build an acyclic reaction network on $\mathcal{A}^{m-q}$, the final network is defined on the initial vertex set and approximates relaxation of $\mathcal{W}$.

To make one step back from $\mathcal{V}^{m}$ let us select the vertices of $\mathcal{A}^{m}$ that are glued cycles from $\mathcal{V}^{m-1}$. Let these vertices be $A_{1}^{m}, A_{2}^{m}, \ldots$. Each $A_{i}^{m}$ corresponds to a glued cycle from $\mathcal{V}^{m-1}, A_{i 1}^{m-1}$ $\rightarrow A_{i 2}^{m-1} \rightarrow \cdots A_{i \tau_{i}}^{m-1} \rightarrow A_{i 1}^{m-1}$, of the length $\tau_{i}$. We assume that the limiting steps in these cycles are $A_{i \tau_{i}}^{m-1} \rightarrow A_{i 1}^{m-1}$. Let us substitute each vertex $A_{i}^{m}$ in $\mathcal{V}^{m}$ by $\tau_{i}$ vertices $A_{i 1}^{m-1}, A_{i 2}^{m-1}, \ldots A_{i \tau_{i}}^{m-1}$ and add to $\mathcal{V}^{m}$ reactions $A_{i 1}^{m-1} \rightarrow A_{i 2}^{m-1} \rightarrow \cdots A_{i \tau_{i}}^{m-1}$ (that are the cycle reactions without the limiting step) with corresponding constants from $\mathcal{V}^{m-1}$.

If there exists an outgoing reaction $A_{i}^{m} \rightarrow B$ in $\mathcal{V}^{m}$ then we substitute it by the reaction $A_{i \tau_{i}}^{m-1} \rightarrow B$ with the same constant, i.e. outgoing reactions $A_{i}^{m} \rightarrow \cdots$ are reattached to the heads of the limiting steps (Fig. 6). Let us rearrange reactions from $\mathcal{V}^{m}$ of the form 


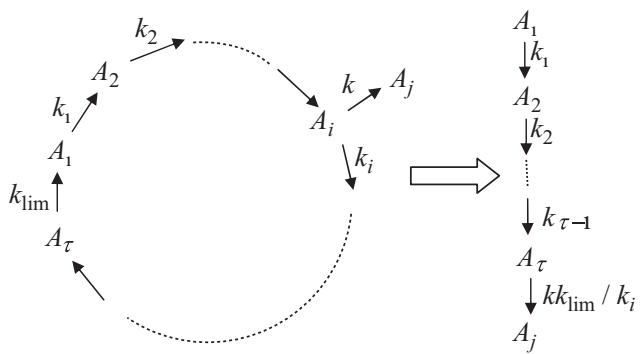

Fig. 6. The main operation of the cycle surgery: on a step back we get a cycle $A_{1} \rightarrow \cdots \rightarrow A_{\tau} \rightarrow A_{1}$ with the limiting step $A_{\tau} \rightarrow A_{1}$ and one outgoing reaction $A_{i} \rightarrow A_{j}$. We should delete the limiting step, reattach ("recharge") the outgoing reaction $A_{i} \rightarrow A_{j}$ from $A_{i}$ to $A_{\tau}$ and change its rate constant $k$ to the rate constant $k k_{\text {lim }} / k_{i}$. The new value of reaction rate constant is always smaller than the initial one: $k k_{\lim } / k_{i}<k$ if $k_{\lim } \neq k_{i}$. For this operation only one condition $k \ll k_{i}$ is necessary $(k$ should be small with respect to reaction $A_{i} \rightarrow A_{i+1}$ rate constant, and can exceed any other reaction rate constant).

$B \rightarrow A_{i}^{m}$. These reactions have prototypes in $\mathcal{V}^{m-1}$ (before the last gluing). We simply restore these reactions. If there exists a reaction $A_{i}^{m} \rightarrow A_{j}^{m}$ then we find the prototype in $\mathcal{V}^{m-1}, A \rightarrow B$, and substitute the reaction by $A_{i \tau_{i}}^{m-1} \rightarrow B$ with the same constant, as for $A_{i}^{m} \rightarrow A_{j}^{m}$.

After that step is performed, the vertices set is $\mathcal{A}^{m-1}$, but the reaction set differs from the reactions of the network $\mathcal{V}^{m-1}$ : the limiting steps of cycles are excluded and the outgoing reactions of glued cycles are included (reattached to the heads of the limiting steps). To make the next step, we select vertices of $\mathcal{A}^{m-1}$ that are glued cycles from $\mathcal{V}^{m-2}$, substitute these vertices by vertices of cycles, delete the limiting steps, attach outgoing reactions to the heads of the limiting steps, and for incoming reactions restore their prototypes from $\mathcal{V}^{m-2}$, and so on.

After all, we restore all the glued cycles, and construct an acyclic reaction network on the set $\mathcal{A}$. This acyclic network approximates relaxation of the network $\mathcal{W}$. We call this system the dominant system of $\mathcal{W}$ and use notation $\operatorname{dom} \bmod (\mathcal{W})$.

In the simplest case, the dominant system is determined by the ordering of constants. But for sufficiently complex systems we need to introduce auxiliary elementary reactions. They appear after cycle gluing and have monomial rate constants of the form $k_{\zeta}=\prod_{i} k_{i}^{\varsigma_{i}}$, where $s_{i}$ are integers, but not mandatory positive. The dominant system depends on the place of these monomial values among the ordered constants. For systems with well separated constants we can also assume that each of these new constants will be well separated from other constants (Gorban and Radulescu, 2008).

\subsection{Example}

To demonstrate a possible branching of described algorithm for cycles surgery (gluing, restoring and cutting) with necessity of additional orderings, let us consider the following system:

$A_{1} \stackrel{1}{\rightarrow} A_{2} \stackrel{6}{\rightarrow} A_{3} \stackrel{2}{\rightarrow} A_{4} \stackrel{3}{\rightarrow} A_{5} \stackrel{4}{\rightarrow} A_{3}, \quad A_{4} \stackrel{5}{\rightarrow} A_{2}$

where the upper index marks the order of rate constants. The auxiliary discrete dynamical system for reaction network (31) is

$A_{1} \stackrel{1}{\rightarrow} A_{2} \stackrel{6}{\rightarrow} A_{3} \stackrel{2}{\rightarrow} A_{4} \stackrel{3}{\rightarrow} A_{5} \stackrel{4}{\rightarrow} A_{3}$.

It has only one attractor, a cycle $A_{3} \stackrel{2}{\rightarrow} A_{4} \stackrel{3}{\rightarrow} A_{5} \stackrel{4}{\rightarrow} A_{3}$. This cycle is not a sink for the whole network (31) because reaction $A_{4} \stackrel{5}{\rightarrow} A_{2}$ leads from that cycle. After gluing the cycle into a vertex $A_{3}^{1}$ we get the new network $A_{1} \stackrel{1}{\rightarrow} A_{2} \stackrel{g}{\rightarrow} A_{3}^{1} \stackrel{?}{\rightarrow} A_{2}$. The rate constant for the reaction $A_{3}^{1} \rightarrow A_{2}$ is $k_{23}^{1}=k_{24} k_{35} / k_{54}$, where $k_{i j}$ is the rate constant for the reaction $A_{j} \rightarrow A_{i}$ in the initial network ( $k_{35}$ is the cycle limiting reaction). The new network coincides with its auxiliary system and has one cycle, $A_{2} \stackrel{6}{\rightarrow} A_{3}^{1} \stackrel{?}{\rightarrow} A_{2}$. This cycle is a sink, hence, we can start the back process of cycles restoring and cutting. One question arises immediately: which constant is smaller, $k_{32}$ or $k_{23}^{1}$. The smallest of them is the limiting constant, and the answer depends on this choice. Let us consider two possibilities separately: (1) $k_{32}>k_{23}^{1}$ and (2) $k_{32}<k_{23}^{1}$.

(1) Let as assume that $k_{32}>k_{23}^{1}$. The final auxiliary system after

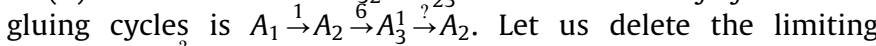
reaction $A_{3}^{1} \stackrel{?}{\rightarrow} A_{2}$ from the cycle. We get an acyclic system $A_{1} \underset{3}{\stackrel{1}{\rightarrow}} A_{2} \underset{4}{\stackrel{6}{\rightarrow}} A_{3}^{1}$. The component $A_{3}^{1}$ is the glued cycle $A_{3} \stackrel{2}{\rightarrow}$ $A_{4} \stackrel{3}{\rightarrow} A_{5} \stackrel{4}{\rightarrow} A_{3}$. Let us restore this cycle and delete the limiting reaction $A_{5} \stackrel{4}{\rightarrow} A_{3}$. We get the dominant system $A_{1} \stackrel{1}{\rightarrow} A_{2}$ $\stackrel{6}{\rightarrow} A_{3} \stackrel{2}{\rightarrow} A_{4} \stackrel{3}{\rightarrow} A_{5}$. Relaxation of this system approximates relaxation of the initial network (31) under additional condition $k_{32}>k_{23}^{1}$.

(2) Let as assume now that $k_{32}<k_{23}^{1}$. The final auxiliary system after gluing cycles is the same, $A_{1} \stackrel{1}{\rightarrow} A_{2} \stackrel{6}{\rightarrow} A_{3}^{1} \stackrel{?}{\rightarrow} A_{2}$, but the limiting step in the cycle is different, $A_{2} \stackrel{6}{\rightarrow} A_{3}^{1}$. After cutting this step, we get acyclic system $A_{1} \stackrel{1}{\rightarrow} A_{2} \leftarrow ? A_{3}^{1}$, where the last reaction has rate constant $k_{23}^{1}$.

The component $A_{3}^{1}$ is the glued cycle

$A_{3} \stackrel{2}{\rightarrow} A_{4} \stackrel{3}{\rightarrow} A_{5} \stackrel{4}{\rightarrow} A_{3}$.

Let us restore this cycle and delete the limiting reaction $A_{5} \stackrel{4}{\rightarrow} A_{3}$. The connection from glued cycle $A_{3}^{1} \stackrel{?}{\rightarrow} A_{2}$ with constant $k_{23}^{1}$ transforms into connection $A_{5} \stackrel{?}{\rightarrow} A_{2}$ with the same constant $k_{23}^{1}$.

We get the dominant system:

$A_{1} \stackrel{1}{\rightarrow} A_{2}, \quad A_{3} \stackrel{2}{\rightarrow} A_{4} \stackrel{3}{\rightarrow} A_{5} \stackrel{?}{\rightarrow} A_{2}$.

The order of constants is now known: $k_{21}>k_{43}>k_{54}>k_{23}^{1}$, and we can substitute the sign "?" by " 4 ": $A_{3} \stackrel{2}{\rightarrow} A_{4} \stackrel{3}{\rightarrow} A_{5} \stackrel{4}{\rightarrow} A_{2}$.

For both cases, $k_{32}>k_{23}^{1}\left(k_{23}^{1}=k_{24} k_{35} / k_{54}\right)$ and $k_{32}<k_{23}^{1}$ it is easy to find the eigenvectors explicitly and to write the solution to the kinetic equations in explicit form.

\section{The reversible triangle of reactions}

In this section, we illustrate the analysis of dominant systems on a simple example, the reversible triangle of reactions.

$A_{1} \leftrightarrow A_{2} \leftrightarrow A_{3} \leftrightarrow A_{1}$

This triangle appeared in many works as an ideal object for a case study. Our favorite example is the work of Wei and Prater (1962). Now in our study the triangle (32) is not necessarily a closed system. We can assume that it is a subsystem of a larger system, and any reaction $A_{i} \rightarrow A_{j}$ represents a reaction of the form $\cdots+A_{i} \rightarrow A_{j}+\cdots$, where unknown but slow components are substituted by dots. This means that there are no mandatory relations between reaction rate constants, and six reaction rate constants are arbitrary non-negative numbers.

Let the reaction rate constant $k_{21}$ for the reaction $A_{1} \rightarrow A_{2}$ be the largest.

Let us describe all possible auxiliary dynamical systems for the triangle (32). For each vertex, we have to select the fastest outgoing reaction. For $A_{1}$, it is always $A_{1} \rightarrow A_{2}$, because of our choice of enumeration (the higher scheme in Fig. 7). There exist two choices of the fastest outgoing reaction for two other vertices and, therefore, only four versions of auxiliary dynamical systems for (32) (Fig. 7). Let us analyze in detail case (a). For the cases (b) and (c) the details of computations are similar. The irreversible cycle (d) is even simpler and was already discussed.

\subsection{Auxiliary system (a): $A_{1} \leftrightarrow A_{2} \leftarrow A_{3} ; k_{12}>k_{32}, k_{23}>k_{13}$}

\subsubsection{Gluing cycles}

The attractor is a cycle (with only two vertices) $A_{1} \leftrightarrow A_{2}$. This is not a sink, because two outgoing reactions exist: $A_{1} \rightarrow A_{3}$ and 


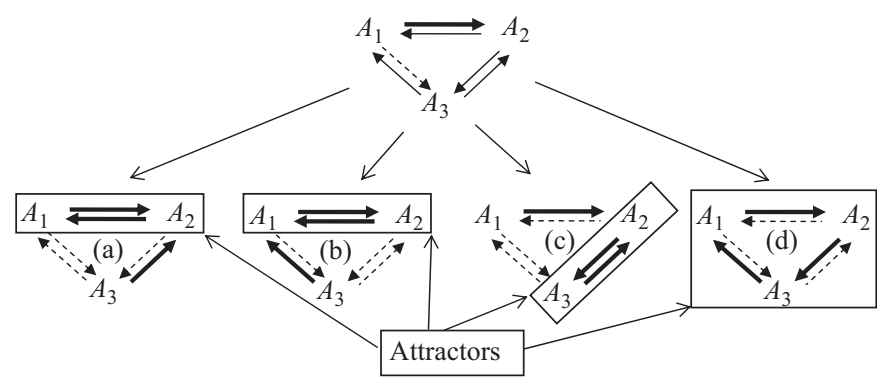

Fig. 7. Four possible auxiliary dynamical systems for the reversible triangle of reactions with $k_{21}>k_{i j}$ for $(i, j) \neq(2,1)$ : (a) $k_{12}>k_{32}, k_{23}>k_{13}$; (b) $k_{12}>k_{32}$, $k_{13}>k_{23}$; (c) $k_{32}>k_{12}, k_{23}>k_{13}$; (d) $k_{32}>k_{12}, k_{13}>k_{23}$. For each vertex the outgoing reaction with the largest rate constant is represented by the solid bold arrow, and other reactions are represented by the dashed arrows. The digraphs formed by solid bold arrows are the auxiliary discrete dynamical systems. Attractors of these systems are isolated in frames.

$A_{2} \rightarrow A_{3}$. They are relatively slow: $k_{31} \ll k_{21}$ and $k_{32} \ll k_{12}$. The limiting step in this cycle is $A_{2} \rightarrow A_{1}$ with the rate constant $k_{12}$. We have to glue the cycle $A_{1} \leftrightarrow A_{2}$ into one new component $A_{1}^{1}$ and to add a new reaction $A_{1}^{1} \rightarrow A_{3}$ with the rate constant (see Fig. 5)

$k_{31}^{1}=\max \left\{k_{32}, k_{31} k_{12} / k_{21}\right\}$.

As a result, we get a new system, $A_{1}^{1} \leftrightarrow A_{3}$ with reaction rate constants $k_{31}^{1}$ (for $A_{1}^{1} \rightarrow A_{3}$ ) and initial $k_{23}$ (for $A_{1}^{1} \leftarrow A_{3}$ ). This cycle is a sink, because it has no outgoing reactions (the whole system is a trivial example of a sink).

\subsubsection{Dominant system}

At the next step, we have to restore and cut the cycles. First cycle to cut is the result of cycle gluing, $A_{1}^{1} \leftrightarrow A_{3}$. It is necessary to delete the limiting step, i.e. the reaction with the smallest rate constant. If $k_{31}^{1}>k_{23}$, then we get $A_{1}^{1} \rightarrow A_{3}$. If, inverse, $k_{23}>k_{31}^{1}$, then we obtain $A_{1}^{1} \leftarrow A_{3}$.

After that, we have to restore and cut the cycle which was glued into the vertex $A_{1}^{1}$. This is the two-vertices cycle $A_{1} \leftrightarrow A_{2}$. The limiting step for this cycle is $A_{1} \leftarrow A_{2}$, because $k_{21} \gg k_{12}$. If $k_{31}^{1}>k_{23}$, then following the rule visualized by Fig. 6 , we get the dominant system $A_{1} \rightarrow A_{2} \rightarrow A_{3}$ with reaction rate constants $k_{21}$ for $A_{1} \rightarrow A_{2}$ and $k_{31}^{1}$ for $A_{2} \rightarrow A_{3}$. If $k_{23}>k_{31}^{1}$ then we obtain $A_{1} \rightarrow A_{2} \leftarrow A_{3}$ with reaction rate constants $k_{21}$ for $A_{1} \rightarrow A_{2}$ and $k_{23}$ for $A_{2} \leftarrow A_{3}$. All the procedure is illustrated by Fig. 8 .

\subsubsection{Eigenvalues and eigenvectors}

The eigenvalues and the corresponding eigenvectors for dominant systems in case (a) are represented below in zero-one asymptotic.

(i) $k_{31}^{1}>k_{23}$,

the dominant system $A_{1} \rightarrow A_{2} \rightarrow A_{3}$ :

$\lambda_{0}=0, \quad r^{0} \approx(0,0,1), \quad l^{0}=(1,1,1)$,

$\lambda_{1} \approx-k_{21}, \quad r^{1} \approx(1,-1,0), \quad l^{1} \approx(1,0,0)$,

$\lambda_{2} \approx-k_{31}^{1}, \quad r^{2} \approx(0,1,-1), \quad l^{2} \approx(1,1,0)$.

(ii) $k_{23}>k_{31}^{1}$,

the dominant system $A_{1} \rightarrow A_{2} \leftarrow A_{3}$ :

$\lambda_{0}=0, \quad r^{0} \approx(0,1,0), \quad l^{0}=(1,1,1)$,

$\lambda_{1} \approx-k_{21}, \quad r^{1} \approx(1,-1,0), \quad l^{1} \approx(1,0,0)$,

$\lambda_{2} \approx-k_{23}, \quad r^{2} \approx(0,-1,1), \quad l^{2} \approx(0,0,1)$.

Here, the value of $k_{31}^{1}$ is given by formula (33).

Analysis of examples provided us by an important conclusion: the number of different dominant systems in examples was less

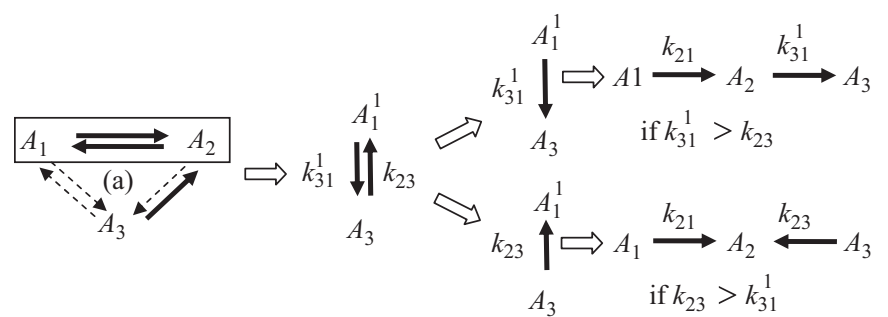

Fig. 8. Dominant systems for case (a) (defined in Fig. 7).

than the number of all possible orderings. For many pairs of constants $k_{i j}, k_{l r}$ it is not important which of them is larger. There is no need to consider all orderings of monomials. We have to consider only those inequalities between constants and monomials that appear in the construction of the dominant systems.

\section{Corrections to dominant dynamics}

The hierarchy of systems $\mathcal{W}, \mathcal{W}^{1}, \mathcal{W}^{2}, \ldots$ can be used for multigrid correction of the dominant dynamics. The simple example of multigrid approach gives the algorithm of steady state approximation (Gorban and Radulescu, 2008). For this purpose, on the way up (cycle restoration and cutting, Section 5.3.2) we calculate distribution in restoring cycles with higher accuracy, by exact formula (13), or in linear approximation (15) instead of the simplest zero-one asymptotic (16). Essentially, the way up remains the same.

After termination of the gluing process, we can find all steady state distributions by restoring cycles in the auxiliary reaction network $\mathcal{V}^{m}$. Let $A_{f 1}^{m}, A_{f 2}^{m}, \ldots$ be fixed points of $\Phi^{m}$. The set of steady states for $\mathcal{V}^{m}$ is the set of all distributions on the set of fixed points $\left\{A_{f 1}^{m}, A_{f 2}^{m}, \ldots\right\}$.

Let us take one of the basis distributions, $c_{f i}^{m}=1$, other $c_{i}=0$ on $\mathcal{V}^{m}$. If the vertex $A_{f i}^{m}$ is a glued cycle, then we substitute them by all the vertices of this cycle. Redistribute the concentration $c_{f i}^{m}$ between the vertices of the corresponding cycle by rule (13) (or by an approximation). As a result, we get a set of vertices and a distribution on this set of vertices. If among these vertices there are glued cycles, then we repeat the procedure of cycle restoration. Terminate when there is no glued cycles in the support of the distribution.

The resulting distribution is the approximation to a steady state of $\mathcal{W}$, and the basis of steady states for $\mathcal{W}$ can be approximated by this method.

For example, for the system Fig. 8 we have, first of all, to compute the stationary distribution in the cycle $A_{1}^{1} \leftrightarrow A_{3}, c_{1}^{1}$ and $c_{3}$. On the base of the general formula for a simple cycle (13) we obtain

$w=\frac{1}{\frac{1}{k_{31}^{1}}+\frac{1}{k_{23}}}, \quad c_{1}^{1}=\frac{w}{k_{31}^{1}}, \quad c_{3}=\frac{w}{k_{23}}$.

After that, we have to restore the cycle glued into $A_{1}^{1}$. This means to calculate the concentrations of $A_{1}$ and $A_{2}$ with normalization $c_{1}+c_{2}=c_{1}^{1}$. Formula (13) gives

$w^{\prime}=\frac{c_{1}^{1}}{\frac{1}{k_{21}}+\frac{1}{k_{12}}}, \quad c_{1}=\frac{w^{\prime}}{k_{21}}, \quad c_{2}=\frac{w^{\prime}}{k_{12}}$.

For eigenvectors, there appear two operations of corrections: (i) correction for an acyclic network without branching (43), (45), and (ii) corrections for a cycle with relatively slow outgoing 
reactions (49). These corrections are by-products of the accuracy estimates given in Appendix.

\section{Conclusion}

Now, the idea of limiting step is developed to the asymptotology of multiscale reaction networks. We found the main terms of eigenvectors and eigenvalues asymptotic on logarithmic straight lines $\ln k_{i j}=\theta_{i j} \xi$ when $\xi \rightarrow \infty$. These main terms could be represented by acyclic dominant system which is a piecewise constant function of the direction vectors $\left(\theta_{i j}\right)$. This theory gives the analogue of the Vishik and Ljusternik (1960) theory for chemical reaction networks. We demonstrated also how to construct the accuracy estimates and the first order corrections to eigenvalues and eigenvectors.

There are several ways of using the developed theory and algorithms:

- For direct computation of steady states and relaxation dynamics; this may be useful for complex systems because of the simplicity of the algorithm and resulting formulas and because often we do not know the rate constants for complex networks, and kinetics that is ruled by orderings rather than by exact values of rate constants may be very useful in practically frequent situation when the values of the various reaction constants are unknown or poorly known.

- For planning experiments and mining the experimental data-the observable kinetics is more sensitive to reactions from the dominant network, and much less sensitive to other reactions, the relaxation spectrum of the dominant network is explicitly connected with the correspondent reaction rate constants, and the eigenvectors ("modes") are sensitive to the constant ordering, but not to exact values.

- The steady states and dynamics of the dominant system could serve as a robust first approximation in perturbation theory or as a preconditioning in numerical methods.

The next step should be development of asymptotic estimates for networks with modular structure and time separations between modules, not between individual reactions. But now it seems that the most important further development should be the asymptotology of nonlinear reaction networks. For multiscale nonlinear reaction networks the expected dynamical behavior is to be approximated by the system of dominant networks. These networks may change in time (this is the significant difference from the linear case) but remain relatively simple.

\section{Appendix A. Mathematical backgrounds of accuracy estimation}

\section{A.1. Estimates for perturbed acyclic networks}

The famous Gerschgorin theorem (Marcus and Minc, 1992; Varga, 2004) gives estimates of eigenvalues. We need also estimates of eigenvectors. Below $A=\left(a_{i j}\right)$ is a complex $n \times n$ matrix, $Q_{i}=\sum_{j, j \neq i}$ $\left|a_{j i}\right|$ (sums of non-diagonal elements in columns).

Gerschgorin theorem (Marcus and Minc, 1992, p. 146): The characteristic roots of $A$ lie in the closed region $G^{Q}$ of the $z$-plane

$G^{Q}=\bigcup_{i} G_{i}^{Q}\left(G_{i}^{Q}=\left\{z|| z-a_{i i} \mid \leq Q_{i}\right\}\right.$.

Areas $G_{i}^{Q}$ are the Gerschgorin disks. (The same estimate are valid for sums in rows, $P_{i}$. Here and below we do not duplicate the estimates.)
Gerschgorin disks $G_{i}^{Q}(i=1, \ldots, n)$ are isolated, if $G_{i}^{Q} \cap G_{j}^{Q}=\emptyset$ for $i \neq j$. If disks $G_{i}^{P}(i=1, \ldots, n)$ are isolated, then the spectrum of $A$ is simple, and each Gerschgorin disk $G_{i}^{Q}$ contains one and only one eigenvalue of $A$ (Marcus and Minc, 1992, p. 147).

We assume that Gerschgorin disks $G_{i}^{Q}(i=1, \ldots n)$ are isolated: for all $i, j(i \neq j)$

$\left|a_{i i}-a_{j j}\right|>Q_{i}+Q_{j}$.

Let us introduce the following notations:

$\frac{Q_{i}}{\left|a_{i i}\right|}=\varepsilon_{i}, \quad \varepsilon=\max _{i} \varepsilon_{i}, \quad \frac{\left|a_{i j}\right|}{\left|a_{j j}\right|}=\chi_{i j}, \quad \chi=\max _{i, j, i \neq j} \chi_{i j}$,

$g_{i}=\min _{j, j \neq i} \frac{\left|a_{i i}-a_{j j}\right|}{\left|a_{i i}\right|}, \quad g=\operatorname{ming}_{i}$.

Usually, we consider $\varepsilon_{i}$ and $\chi_{i j}$ as sufficiently small numbers. In contrary, the diagonal gap g should not be small, (this is the gap condition). For example, if for any two diagonal elements $a_{i i}, a_{j j}$ either $a_{i i} \gg a_{j j}$ or $a_{i i} \ll a_{j j}$, then $g_{i} \gtrsim 1$ for all $i$.

Let $\lambda_{i} \in G_{i}^{Q}$ be the eigenvalue of $A\left(\left|\lambda_{i}-a_{11}\right|<Q_{1}\right)$. Let us estimate the corresponding right eigenvector $r^{(i)}$. We take $r_{i}^{i}=1$ and for $j \neq i$ introduce a $(n-1)$-dimensional vector $\tilde{x}^{i}: \tilde{x}_{j}^{i}=r_{j}^{i}\left(a_{j j}-\right.$ $\left.a_{i i}\right)(i \neq j)$. For $\tilde{x}^{i}$ we get equation

$\left(1-B^{(i)}\right) \tilde{x}^{i}=-\tilde{a}^{i}$

where $\tilde{a}^{i}$ is a vector of the non-diagonal elements of the $i$ th column of $A\left(\tilde{a}_{j}^{i}=a_{i j}, j \neq i\right)$, and the $(n-1) \times(n-1)$ matrix $B^{i}$ has matrix elements $(j, l \neq i)$

$b_{j j}^{(i)}=\frac{\lambda_{i}-a_{i i}}{a_{j j}-a_{i i}}, \quad b_{j l}^{(i)}=\frac{a_{j l}}{a_{l l}-a_{i i}}(l \neq j)$.

Due to the Gerschgorin estimate, $\left|b_{j j}^{(i)}\right|<Q_{i} /\left|a_{j j}-a_{i i}\right|$. From Eq. (41) we obtain

$\tilde{x}^{i}=-\tilde{a}^{i}-B^{(i)}\left(1-B^{(i)}\right)^{-1} \tilde{a}^{i}$.

From this definition and simple estimates in $l^{1}$ norm, we get the following estimate of eigenvectors.

Theorem 2. Let the Gerschgoring disks be isolated, and the diagonal gap be big enough: $g>n \varepsilon$. Then for the ith eigenvector of $A$ the following uniform estimate holds:

$\left|r_{j}^{i}\right| \leq \frac{\chi}{g}+\frac{n \varepsilon^{2}}{g(g-n \varepsilon)} \quad\left(j \neq 1, r_{i}^{i}=1\right)$.

So, if the matrix $A$ is diagonally dominant and the diagonal gap $g$ is big enough, then the eigenvectors are proven to be close to the standard basis vectors with explicit evaluation of accuracy.

The first correction to eigenvectors is also given by Eq. (43). If for the iteration we use the Gerschgorin estimates for eigenvalue $\lambda_{i} \approx a_{i i}$, then we can write in the next approximation for eigenvectors $\left(r_{i}^{i}=1, j \neq i\right)$ :

$r_{j}^{i}=-\frac{a_{j i}}{a_{j j}-a_{i i}}-\frac{\left(B_{\mathrm{nd}}^{(i)}\left(1-B_{\mathrm{nd}}^{(i)}\right)^{-1} \tilde{a}^{i}\right)_{j}}{a_{j j}-a_{i i}}$,

where $B_{\text {nd }}^{(i)}$ is the non-diagonal part of $B^{(i)}$ : it has the same nondiagonal elements and zeros on diagonal. There exists plenty of further simplifications for this iteration formula. For example, one can leave just the first term, that gives the first order approximation in the power of $\varepsilon(\chi \leq \varepsilon)$.

To apply these estimates to an acyclic network supplemented by additional reactions, we have to use the eigenbasis of this acyclic network (Section 4). Direct use of this theorem and estimates for a kinetic matrix $K$ in the standard basis is impossible, the diagonal dominance in this coordinate system is not large, and sums of elements in columns are zero. To apply this theorem we need two lemmas. 
Let $\mathcal{W}$ be a reaction network without branching (a finite dynamical system) with $n$ vertices. Then the number of reactions in $\mathcal{W}$ is $n-f$, where $f$ is the number of fixed points (the vertices without outgoing reactions). Let $\Gamma$ be the set of stoichiometric vectors for $\mathcal{W}$.

Lemma 1. $\Gamma$ forms a basis in the subspace $\left\{c \mid \sum_{i} c_{i}=0\right\}$ if and only if the reaction network $\mathcal{W}$ is acyclic and connected (has only one fixed point).

Let us consider a general reaction network on the set $A_{1}, \ldots, A_{n}$. For stoichiometric vector of reaction $A_{i} \rightarrow A_{l}$ we use notation $\gamma_{l i}$. Assume that the auxiliary dynamical system $i \mapsto \phi(i)$ for a given reaction network is acyclic and has only one attractor, a fixed point. For this auxiliary network, we use notation: $\kappa_{i}=k_{j i}$ for the only reaction $A_{i} \rightarrow A_{j}$, or $\kappa_{i}=0$.

For every reaction of the initial network, $A_{i} \rightarrow A_{l}$, a linear operators $Q_{i l}$ can be defined by its action on the basis vectors, $\gamma_{\phi(i) i}$ :

$Q_{i l}\left(\gamma_{\phi(i)}\right)=\gamma_{l i}, \quad Q_{i l}\left(\gamma_{\phi(p) p}\right)=0 \quad$ for $p \neq i$.

Lemma 2. The kinetic equation for the whole reaction network (9) could be transformed to the form

$$
\begin{aligned}
\frac{\mathrm{d} c}{\mathrm{~d} t} & =\sum_{i}\left(1+\sum_{l, l \neq \phi(i)} \frac{k_{l i}}{\kappa_{i}} Q_{i l}\right) \gamma_{\phi(i) i} \kappa_{i} c_{i} \\
& =\left(1+\sum_{j, l} \frac{k_{l j}}{\kappa_{j}} Q_{j l}\right) \sum_{i} \gamma_{\phi(i) i} \kappa_{i} c_{i} \\
& =\left(1+\sum_{j, l} \frac{k_{l j}}{\kappa_{j}} Q_{j l}\right) \tilde{K} c,
\end{aligned}
$$

where $\tilde{K}$ is kinetic matrix of the kinetic equation for the auxiliary network.

By construction of auxiliary dynamical system, $k_{l i}<\kappa_{i}$ if $l \neq \phi(i)$, and for reaction networks with well separated constants $k_{l i} \ll \kappa_{i}$. Notice also that the matrix $Q_{j l}$ does not depend on rate constants values.

For matrix $\tilde{K}$ we have the eigenbasis in explicit form. Let us represent system (47) in this eigenbasis of $\tilde{K}$. Any matrix $B$ in this eigenbasis has the form $B=\left(\tilde{b}_{i j}\right), \tilde{b}_{i j}=l^{i} B r^{j}=\sum_{q s} l_{q}^{i} b_{q s} r_{s}^{j}$, where $\left(b_{q s}\right)$ is matrix $B$ in the initial basis, $l^{i}$ and $r^{j}$ are left and right eigenvectors of $\tilde{K}$ (27), (28). In eigenbasis of $\tilde{K}$ the estimates of eigenvalues and estimates of eigenvectors are much more efficient than in original coordinates: the system is strongly diagonally dominant. Transformation to this basis is an effective preconditioning for the perturbation theory that uses auxiliary kinetics as a first approximation to the kinetics of the whole system.

\section{A.2. Estimates for perturbed ergodic systems}

Let us consider a strongly connected network with kinetic matrix $K$. The corresponding kinetics is ergodic and there exists unique normalized steady state $c_{i}^{*}>0, \sum_{i} c_{i}^{*}=1$. For each $i$ we define $\kappa_{i}=\sum_{j} k_{j i}$. The number $-\kappa_{i}$ is the $i i$ th diagonal element of unperturbed kinetic matrix $K$.

Let this network be perturbed by outgoing reactions $A_{i} \rightarrow 0$. The perturbation has the "loss form": the perturbed matrix is $K-\operatorname{diag}\left(\varepsilon_{i} \kappa_{i}\right)$, perturbation of each diagonal element is relatively small (diag is the diagonal matrix).

The perturbations $\varepsilon_{i} \kappa_{i}$ are relatively small with respect to $\kappa_{i}$, but not obligatory small with respect to other rate constants.

First, we do not assume anything about value of $\varepsilon_{i} \geq 0$ and make the following transformation. For an arbitrary normalized vector $r\left(r_{i} \geq 0, \sum_{i} r_{i}=1\right)$ we add to the network reactions $A_{i} \rightarrow A_{j}$ with reaction rates $q_{j i}=r_{j} \varepsilon_{i} \kappa_{i}$. We use $Q(r)$ for the kinetic matrix of this additional network. Simple algebra gives

$$
\begin{aligned}
Q(r)+\operatorname{diag}\left(\varepsilon_{i} \kappa_{i}\right) & =\left[\varepsilon_{1} \kappa_{1} r, \varepsilon_{2} \kappa_{2} r, \ldots, \varepsilon_{n} \kappa_{n} r\right] \\
& =r\left(\varepsilon_{1} \kappa_{1}, \varepsilon_{2} \kappa_{2}, \ldots, \varepsilon_{n} \kappa_{n}\right) .
\end{aligned}
$$

Here, in the right hand side we have a matrix, all columns of which are proportional to the vector $r$, this is a product of $r$ on the vector-raw of coefficients. We represent the perturbed matrix in the form $K-\operatorname{diag}\left(\varepsilon_{i} \kappa_{i}\right)=K+Q(r)-\left(Q(r)+\operatorname{diag}\left(\varepsilon_{i} \kappa_{i}\right)\right)$.

Theorem 3. There exists such normalized positive $r^{*}$ that $\left(K+Q\left(r^{*}\right)\right) r^{*}=0$. This $r^{*}$ is an eigenvector of the perturbed network with the eigenvalue $\lambda=\sum_{i} r_{i}^{*} \varepsilon_{i} \kappa_{i}$, and, at the same time, it is a steady-state for the network with kinetic matrix $K+Q\left(r^{*}\right)$.

To prove existence it is sufficient to mention, that for any $r$ the network with kinetic matrix $K+Q(r)$ has unique positive normalized steady state $c^{*}(r)$, which depends continuously on $r$. The map $r \mapsto c^{*}(r)$ has a fixed point $r^{*}$ (the Brouwer fixed point theorem).

This representation allows us to produce useful estimates, for example, when the unperturbed system is a cycle, we find $\mid r_{i}^{*}-$ $c_{i}^{*}|<3 \varepsilon| c_{i}^{*} \mid$ under condition $\varepsilon<0.25$, where $\varepsilon=\sum \varepsilon_{i}$. Formula for the first correction gives $\left(r^{*}=c_{i}^{*}+\delta r_{i}, w=k_{i} c_{i}^{*}\right)$ :

$\delta r_{i}=\frac{v_{i}}{k_{i}}, \quad v_{i}=v+w \sum_{j=1}^{i}\left(\varepsilon c_{j}^{*}-\varepsilon_{j}\right)$

$v=\frac{w}{n} \sum_{i=1}^{n} i\left(\varepsilon c_{i}^{*}-\varepsilon_{i}\right)$

For more complex networks, the explicit formulas for corrections could be produced on the base of the network graphs, similar to the steady-state formulas, presented, for example, by Yablonskii et al. (1991).

So, the asymptotic analysis gives good approximation of eigenvectors and eigenvalues for kinetic matrix. The condition number is big (unbounded) but these estimates work even better when the constants become more separated. Nevertheless, some caution is needed: the error is proven to be small, but the residuals (the values $\|K r-\lambda r\|$ for approximations of $r$ and $\lambda$ ) may be not small (Gorban and Radulescu, 2008).

\section{References}

Andrianov, I.V., Manevitch, L.I., 2002. Asymptotology: Ideas, Methods and Applications (Series: Mathematics and its Applications, vol. 551). Springer, Dordrecht, Boston, London.

Antoulas, A.C., Sorensen, D.C., 2002. The Sylvester equation and approximate balanced reduction. Linear Algebra Appl. 351-352, 671-700.

Aris, R., 1965. Introduction to the Analysis of Chemical Reactors. Prentice-Hall, Inc., Englewood Cliffs, NJ.

Balian, R., Alhassid, Y., Reinhardt, H., 1986. Dissipation in many-body systems: a geometric approach based on information theory. Phys. Rep. 131 (1), 1-146.

Bodenstein, M., 1913. Eine theorie der photochemischen reaktionsgeschwindigkeiten. Z . Phys. Chem. 85, 329-397.

Boyd, R.K., 1978. Some common oversimplifications in teaching chemical kinetics. J. Chem. Educ. 55, 84-89.

Brown, G.C., Cooper, C.E., 1993. Control analysis applied to a single enzymes: can an isolated enzyme have a unique rate-limiting step?. Biochem. J. 294, 87-94.

Bykov, V., Goldfarb, I., Gol'dshtein, V., Maas, U., 2006. On a modified version of ILDM approach: asymptotical analysis based on integral manifolds method. IMA J. Appl. Math. 71 (3), 359-382.

Christiansen, J.A., 1953. The elucidation of reaction mechanisms by the method of intermediates in quasi-stationary concentrations. Adv. Catal. 5, 311-353.

Condon, M., Ivanov, R., 2004. Empirical balanced truncation of nonlinear systems. J. Nonlinear Sci. 14, 405-414.

Cornish-Bowden, A., Cardenas, M.L., 1990. Control on Metabolic Processes. Plenum Press, New York.

Coxson, P.G., Bischoff, K.B., 1987. Lumping strategy 2 system theoretic approach. Ind. Eng. Chem. Res. 26 (10), 2151-2157.

Djouad, R., Sportisse, B., 2002. Partitioning techniques and lumping computation for reducing chemical kinetics. APLA: an automatic partitioning and lumping algorithm. Appl. Num. Math. 43 (4), 383-398. 
Dobrushin, R.L., 1956. Central limit theorem for non-stationary Markov chains I, II. Theor. Prob. Appl. 1, 163-180 (329-383).

Dokoumetzidis, A. Aarons, L., 2009. Proper lumping in systems biology models. IET Syst. Biol. 3 (1), 40-51.

Farkas, G., 1999. Kinetic lumping schemes. Chem. Eng. Sci. 54 (17), 3909-3915.

Feinberg, M., 1972. On chemical kinetics of a certain class. Arch. Rat. Mech. Anal. 46 (1), $1-41$

Feng, X-J., Hooshangi, S., Chen, D., Li, G., Weiss, R., Rabitz, H., 2004. Optimizing genetic circuits by global sensitivity analysis. Biophys. J. 87, 2195-2202.

Gibbs, G.W., 1902. Elementary Principles in Statistical Mechanics. Yale University Press, New Haven.

Golub, G.H., Van Loan, C.F., 1996. Matrix Computations, third ed The Johns Hopkins University Press, Baltimore.

Gorban, A.N., 1984. Equilibrium Encircling. Equations of Chemical Kinetics and their Thermodynamic Analysis. Nauka, Novosibirsk.

Gorban, A.N., Bykov, V.I., Yablonskii, G.S., 1986a. Thermodynamic function analogue for reactions proceeding without interaction of various substances. Chem. Eng. Sci. 41 (11), 2739-2745.

Gorban, A.N., Bykov, V.I., Yablonskii, G.S., 1986b. Essays on Chemical Relaxation. Nauka, Novosibirsk.

Gorban, A.N., Karlin, I.V., 2003. Method of invariant manifold for chemical kinetics. Chem. Eng. Sci. 58, 4751-4768.

Gorban, A.N., Karlin, I.V., 2005. Invariant manifolds for physical and chemica kinetics. In: Lecture Notes in Physics, vol. 660. Springer, Berlin, Heidelberg New York.

Gorban, A.N., Karlin, I.V., Ilg, P., Öttinger, H.C., 2001. Corrections and enhancements of quasi-equilibrium states. J. Non-Newtonian Fluid Mech. 96 (2001), 203-219.

Gorban, A.N., Karlin, I.V., Zinovyev, A.Yu., 2004. Invariant grids for reaction kinetics. Physica A 333 (2004), 106-154 Preprint online: <http://www.ihes.fr/PRE PRINTS/P03/Resu/resu-P03-42.html $>$.

Gorban, A.N., Radulescu, O., 2008. Dynamic and static limitation in reaction networks revisited. Adv. Chem. Eng. 34, 103-173 e-print: 〈http://arxiv.org/abs/ physics/0703278>

Greuel, G.-M., Pfister, G., 2002. A Singular Introduction to Commutative Algebra. Springer, Berlin, Heidelberg, New York.

Gugercin, S., Antoulas, A.C., 2004. A survey of model reduction by balanced truncation and some new results. Int. J. Control 77 (8), 748-766.

Hangos, K.M., Bokor, J., Szederkényi, G., 2004. Analysis and Control of Nonlinear Process Systems. Springer, London.

Hangos, K.M., Cameron, I.T., 2001. Process Modelling and Model Analysis. Academic Press, London.

Helfferich, F.G., 1989. Systematic approach to elucidation of multistep reaction networks. J. Phys. Chem. 93 (18), 6676-6681.

Hutchinson, P., Luss, D., 1970. Lumping of mixtures with many parallel first order reactions. Chem. Eng. J. 1, 129-135.

Jaynes, E.T., 1963. Information theory and statistical mechanics. In: Ford, K.W. (Ed.) Statistical Physics. Brandeis Lectures, vol. 3. Benjamin, New York, pp. 160-185.

Johnston, H.S., 1966. Gas Phase Reaction Theory. Roland Press, New York.

Kazantzis, N., Kravaris, C., 2006. A new model reduction method for nonlinear dynamical systems using singular PDE theory. In: Gorban, A.N., Kazantzis, N., Kevrekidis, Y.G., Ottinger, H.C., Theodoropoulos, C. (Eds.), Model Reduction and Coarse-Graining Approaches for Multiscale Phenomena. Springer, Berlin, pp. 3-15.

Klonowski, W., 1983. Simplifying principles for chemical and enzyme reaction kinetics. Biophys. Chem. 18, 73-87.

Kruskal, M.D., 1963. Asymptotology. In: Dobrot, S. (Ed.), Mathematical Models in Physical Sciences. Prentice-Hall, Englewood Cliffs, NJ, pp. 17-48.

Kuo, J.C., Wei, J., 1969. A lumping analysis in monomolecular reaction systems. Analysis of the approximately lumpable system. Ind. Eng. Chem. Fundam. 8, 124-133.

Lam, S.H., 1993. Using CSP to understand complex chemical kinetics. Combust. Sci. Technol. 89 (5), 375-404.

Lall, S., Marsden, J.E., Glavaki, S., 2002. A subspace approach to balanced truncation for model reduction of nonlinear control systems. Int. J. Robust Nonlinear Control 12 (6), 519-535.

Lam, S.H., Goussis, D.A., 1994. The CSP method for simplifying kinetics. Int. J. Chem. Kinetics 26, 461-486.

Li, G., Rabitz, H., 1989. A general analysis of exact lumping in chemical kinetics. Chem. Eng. Sci. 44, 1413-1430.

Liao, J.C., Lightfoot Jr., E.N., 1988. Lumping analysis of biochemical reaction systems with time scale separation. Biotechnol. Bioeng. 31, 869-879.

Lidskii, V., 1965. Perturbation theory of non-conjugate operators. U.S.S.R. Comput. Math. Math. Phys. 6, 73-85.

Lin, B., Leibovici, C.F., Jorgensen, S.B., 2008. Optimal component lumping: problem formulation and solution techniques. Comput. Chem. Eng. 32, 1167-1172.

Litvinov, G.L., Maslov, V.P. (Eds.), 2005. Idempotent mathematics and mathematica physics. In: Contemporary Mathematics. AMS, Providence.

Maas, U., Pope, S.B., 1992. Simplifying chemical kinetics: intrinsic low-dimensional manifolds in composition space. Combust. Flame 88, 239-264.
Marcus, M., Minc, H., 1992. A Survey of Matrix Theory and Matrix Inequalities. Dover, New York

Maria, G., 2006. Application of lumping analysis in modelling the living systems: a trade-off between simplicity and model quality. Chem. Biochem. Eng. Quart. 20 (4), 353-373.

Meyn, S.R., 2007. Control Techniques for Complex Networks. Cambridge University Press, Cambridge.

Meyn, S.P., Tweedie, R.L., 2009. Markov Chains and Stochastic Stability, second ed Cambridge University Press, Cambridge.

Moore, B.C., 1981. Principal component analysis in linear system: controllability, observability and model reduction. IEEE Trans. Automat. Control AC-26.

Northrop, D.B., 1981. Minimal kinetic mechanism and general equation for deuterium isotope effects on enzymic reactions: uncertainty in detecting a rate-limiting step. Biochemistry 20, 4056-4061.

Northrop, D.B., 2001. Uses of isotope effects in the study of enzymes. Methods 24 117-124.

Pepiot-Desjardins, P., Pitsch, H., 2008. An automatic chemical lumping method for the reduction of large chemical kinetic mechanisms. Combust. Theory Modelling 12 (6), 1089-1108.

Prigogine, I., Defay, R., 1954. Chemical Thermodynamics. Longmans, London.

Procaccia, I., Ross, J., 1977. Stability and relative stability in reactive systems far from equilibrium. I. Thermodynamic analysis. J. Chem. Phys. 67, 5558-5564.

Radulescu, O., Gorban, A., Zinovyev, A., Lilienbaum, A., 2008. Robust simplifications of multiscale biochemical networks. BMC Syst. Biol. 2 (1), $86<$ http://www. biomedcentral.com/1752-0509/2/86 $>$..

Rate-controlling step, 2007. In: IUPAC Compendium of Chemical Terminology, E-version, 〈http://goldbook.iupac.org/R05139.html >.

Ray Jr., W.J., 1983. A rate-limiting step: a quantitative definition. Application to steady-state enzymic reactions. Biochemistry 22, 4625-4637.

Robbiano, L., 1985. Term orderings on the polynomial ring. In: Caviness, B.F. (Ed.), Proceedings of the EUROCAL 85, vol. 2. Lecture Notes in Computer Sciences, vol. 204. Springer, Berlin, Heidelberg, New York, Tokyo, pp. 513-518.

Roussel, M.R., Fraser, S.J., 1991. On the geometry of transient relaxation. J. Chem. Phys. 94, 7106-7111.

Segel, L.A., Slemrod, M., 1989. The quasi-steady-state assumption: a case study in perturbation. SIAM Rev. 31, 446-477.

Semenov, N.N., 1939. On the kinetics of complex reactions. J. Chem. Phys. 7, 683-699.

Seneta, E., 1981. Nonnegative Matrices and Markov Chains. Springer, New York.

Stueckelberg, E.C.G., 1952. Theoreme $H$ et unitarite de S. Helv. Phys. Acta 25 (5) 577-580.

Temkin, O.N., Zeigarnik, A.V., Bonchev, D.G., 1996. Chemical Reaction Networks: A Graph-Theoretical Approach. CRC Press, Boca Raton, FL.

Toth, J., Li, G., Rabitz, H., Tomlin, A.S., 1997. The effect of lumping and expanding on kinetic differential equations. SIAM J. Appl. Math. 57, 1531-1556.

Turanyi, T., Tomlin, A.S., Pilling, M.J., 1993. On the error of the quasi-steady-state approximation. J. Phys. Chem. 97 (1), 163-172.

Van Mieghem, P., 2006. Performance Analysis of Communications Networks and Systems. Cambridge University Press, Cambridge.

Varga, R.S., 2004. Gerschgorin and His Circled, Springer Series in Computational Mathematics, vol. 36. Springer, Berlin, Heidelberg, New York.

Vishik, M.I., Ljusternik, L.A., 1960. Solution of some perturbation problems in the case of matrices and self-adjoint or non-self adjoint differential equations. I. Russian Math. Surveys 15, 1-73.

Vora, N., Daoutidis, P., 2001. Nonlinear model reduction of chemical reaction systems. A.I.Ch.E. J. 47 (10), 2320-2332.

Wei, J., Prater, C., 1962. The structure and analysis of complex reaction systems. Adv. Catal. 13, 203-393.

Wei, J., Kuo, J.C., 1969. A lumping analysis in monomolecular reaction systems: analysis of the exactly lumpable system. Ind. Eng. Chem. Fundam. 8, 114-123.

White, R.B., 2006. Asymptotic Analysis of Differential Equations. Imperial College Press \& World Scientific, London.

Whitehouse, L.E., Tomlin, A.S., Pilling, M.J., 2004. Systematic reduction of complex tropospheric chemical mechanisms, part II: lumping using a time-scale based approach. Atmos. Chem. Phys. 4, 2057-2081.

Yablonskii, G.S., Bykov, V.I., Gorban, A.N., Elokhin, V.I., 1991. Kinetic models of catalytic reactions. In: Compton, R.G. (Ed.), Comprehensive Chemical Kinetics, vol. 32. Elsevier, Amsterdam.

Yablonsky, G.S., Mareels, I.M.Y., Lazman, M., 2003. The principle of critical simplification in chemical kinetics. Chem. Eng. Sci. 58, 4833-4842.

Yablonsky, G.S., Olea, M., Marin, G.B., 2003. Temporal analysis of products (TAP): basic principles, applications and theory. J. Catal. 216, 120-134.

Zagaris, A., Kaper, H.G., Kaper, T.J., 2004. Analysis of the computational singular perturbation reduction method for chemical kinetics. J. Nonlinear Sci. 14, $59-91$.

Zavala, C.D., Rodriguez, J.E.R., Vargas-Villamil, F.D., 2004. An algorithm for pseudocompound delumping and lumping into homologous groups. Petrol. Sci. Technol. 22 (1-2), 45-60. 